\title{
Catalytic Asymmetric Reductive Michael Cyclization
}

\author{
Jung Woon Yang, Maria T. Hechavarria Fonseca, and Benjamin List* \\ Max-Planck-Institut für Kohlenforschung, D-45470 Mülheim an der Ruhr, Germany, \\ list@mpi-muelheim.mpg.de
}

\section{Supporting Information}

General: Reactions were monitored by thin layer chromatography using $0.25 \mathrm{~mm}$ E. Merck silica gel coated glass plates (60F-254) using UV light to visualize the course of reaction. Flash column chromatography was performed using E. Merck siliga gel (60, particle size $0.040-0.063 \mathrm{~mm}$ ). Chemical yields refer to pure isolated substances. ${ }^{1} \mathrm{H}$ and ${ }^{13} \mathrm{C}$ NMR spectra were obtained using either a Bruker DPX-300 or AV-400 spectrometer. Chemical shifts are reported in ppm from tetramethylsilane with the solvent resonance as the internal standard. The following abbreviations were used to designate chemical shift mutiplicities: $\mathrm{s}=$ singlet, $\mathrm{d}$ = doublet, $\mathrm{t}=$ triplet, $\mathrm{q}=$ quartet, $\mathrm{h}=$ heptet, $\mathrm{m}=$ multiplet, $\mathrm{br}=$ broad. $\mathrm{MS}(\mathrm{EI})$ : Finnigan MAT 8200 (70 eV), ESI-MS: Finnigan MAT 95, accurate mass determinations: Bruker APEX III FT-MS (7 T magnet).

\section{Preparation of Catalysts}

Catalysts 9e-f were prepared according to the procedure of MacMillan et al. ${ }^{1}$<smiles>CN1C(=O)[C@@H](Cc2ccc(Br)cc2)N[C@@H]1C(C)(C)C</smiles>

9e (free base)

${ }^{1} \mathrm{H}$ NMR (400 MHz, $\left.\mathrm{CDCl}_{3}\right) \delta$ 7.41-7.26 (m, 5H), 7.16 (d, $J=$ $8.6 \mathrm{~Hz}, 2 \mathrm{H}), 6.89$ (d, $J=8.6 \mathrm{~Hz}, 2 \mathrm{H}), 5.02$ (s, 2H), 4.04 (s, 1H), 3.67-3.64 (m, 1H), 3.06 (dd, $J=14.0,4.1 \mathrm{~Hz}, 1 \mathrm{H}), 2.92$ (dd, $J=$ 13.8, 7.0 Hz, 1H), 2.88 (s, 3H), 1.82 (br, 1H), 0.81 (s, 9H); ${ }^{13} \mathrm{C}$ NMR (100 MHz, $\left.\mathrm{CDCl}_{3}\right) \delta 175.0,157.3,136.7,130.3,129.6,128.2,127.5,127.0,114.7,82.1$, 69.7, 59.1, 36.7, 34.6, 30.3, 25.0; HRMS (ESI) calcd for $\mathrm{MNa}^{+}$375.204299, found 375.204124.

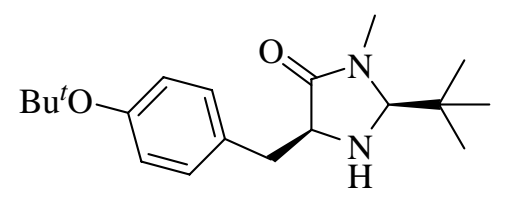

9f (free base)

${ }^{1} \mathrm{H}$ NMR (300 MHz, $\left.\mathrm{CDCl}_{3}\right) \delta 7.07$ (d, $\left.J=8.4 \mathrm{~Hz}, 2 \mathrm{H}\right), 6.85$ (d, $J=8.6 \mathrm{~Hz}, 2 \mathrm{H}), 3.98$ (s, 1H), 3.63-3.60 (m, 1H), 2.98 (d, $J=$ $5.3 \mathrm{~Hz}, 2 \mathrm{H}), 2.83$ (s, 3H), 1.24 (s, 9H), 0.73 (s, 9H); ${ }^{13} \mathrm{C}$ NMR

\footnotetext{
${ }^{1}$ Paras, N. A.; MacMillan, D. W. C. J. Am. Chem. Soc. 2002, 124, 7894 - 7895
} 
$\left(100 \mathrm{MHz}, \mathrm{CDCl}_{3}\right) \delta 175.1,153.8,131.8,129.8,124.0,82.1,77.9,58.9,36.3,34.5,30.3,28.4$, 25.0; HRMS (ESI) calcd for $\mathrm{MNa}^{+}$341.219949, found 341.219842.

\section{General procedure for the preparation of enal enones, ${ }^{2}$ illustrated with compound 10}

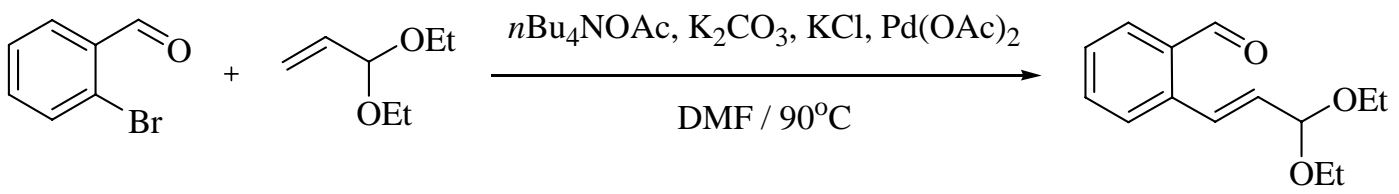

To a stirred solution of 2-bromobenzaldehyde (6.14 g, $33.17 \mathrm{mmol})$ in $40 \mathrm{~mL}$ of DMF were added acrolein diethyl acetal (8.64 g, $66.34 \mathrm{mmol}$ ), $n \mathrm{Bu}_{4} \mathrm{NOAc}$ (20.0 g, $66.34 \mathrm{mmol}$ ), $\mathrm{K}_{2} \mathrm{CO}_{3}$ (6.88 g, $49.76 \mathrm{mmol}), \mathrm{KCl}$ (2.47 g, $33.17 \mathrm{mmol})$, and Pd(OAc) 2 (223.4 g, $0.995 \mathrm{mmol})$. The mixture was stirred at $90{ }^{\circ} \mathrm{C}$ for $2 \mathrm{~h}$. After cooling, the reaction mixture was quenched using saturated aqueous $\mathrm{NaCl}$ and extracted with diethyl ether. The combined organic layer were dried $\left(\mathrm{Na}_{2} \mathrm{SO}_{4}\right)$, filtered, and concentrated in vacuo. The residue was purified by flash chromatography on silica gel (10\% diethyl ether/hexane) to give $4.08 \mathrm{~g}$ (52\% yield) of the unsaturated acetal.

${ }^{1} \mathrm{H}$ NMR (400 MHz, $\mathrm{CDCl}_{3}$ ) $\delta 10.2$ (s, 1H), 7.76 (d, $\left.J=0.7 \mathrm{~Hz}, 1 \mathrm{H}\right), 7.49$ (m, 2H), 7.36 (m, 1H), 6.07 (dd, $J=15.9,5.1 \mathrm{~Hz}, 1 \mathrm{H}), 5.03(\mathrm{~m}, 1 \mathrm{H}), 3.66$ (m, 3H), $3.52(\mathrm{~m}, 3 \mathrm{H}), 1.17(\mathrm{t}, J=5.0$ $\mathrm{Hz}, 6 \mathrm{H}) ;{ }^{13} \mathrm{C}$ NMR $\left(100 \mathrm{MHz}, \mathrm{CDCl}_{3}\right) \delta$ 191.8, 134.2, 132.7, 131.8, 131.1, 128.7, 127.6, 127.3, 100.9, 61.0, 14.9 .
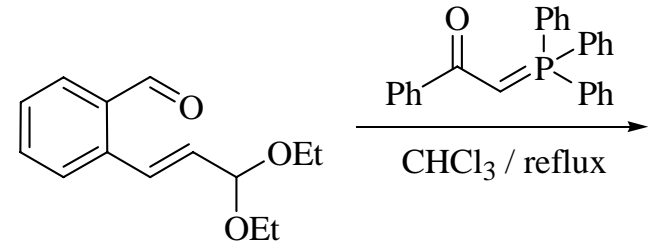<smiles>CCOC(/C=C/c1ccccc1/C=C/C(=O)c1ccccc1)OCC</smiles>

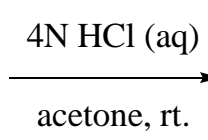

acetone, rt.<smiles>O=C/C=C/c1ccccc1/C=C/C(=O)c1ccccc1</smiles>

10

To a solution of the aldehyde $(1.60 \mathrm{~g}, 6.83 \mathrm{mmol})$ in $50 \mathrm{~mL}$ of $\mathrm{CHCl}_{3}$ was added (benzoylmethylene)triphenylphosphorane (3.90 g, $10.25 \mathrm{mmol})$ at room temperature. The reaction mixture was stirred overnight under reflux condition. Then, the solvent was removed under reduced pressure and the crude material was purified by flash chromatography (10\% ethyl acetate/hexane), providing the corresponding enone (1.93 g, 84\%) as a brownish oil. Subsequently, to a stirred solution of the enone $(1.80 \mathrm{~g}, 5.35 \mathrm{mmol})$ in $53.5 \mathrm{~mL}$ of acetone was added aqueous $4 \mathrm{~N} \mathrm{HCl}(5.35 \mathrm{~mL})$ solution at room temperature. After $10 \mathrm{~min}$, the reaction mixture was quenched using saturated aqueous $\mathrm{NaCl}$ and extracted with diethyl ether. The combined organic layer were dried $\left(\mathrm{Na}_{2} \mathrm{SO}_{4}\right)$, filtered and concentrated in vacuo. The

\footnotetext{
${ }^{2}$ Battistuzzi, G.; Cacchi, S.; Fabrizi, G. Org. Lett., 2003, 5, 777-780.
} 
residue was recrystallized from dichloromethane/diethylether to give $1.25 \mathrm{~g}$ (89\%) of $\mathbf{1 0}$ as yellowish solid. Compound 12, 14, 16, 18, 20, 22 (Knoevenagel instead of Wittig) were prepared analogously.

${ }^{1} \mathrm{H}$ NMR (300 MHz, $\left.\mathrm{CDCl}_{3}\right) \delta 9.71$ (d, $\left.J=7.6 \mathrm{~Hz}, 1 \mathrm{H}\right), 8.14$ (d, $\left.J=15.4 \mathrm{~Hz}, 1 \mathrm{H}\right), 7.97$ (d, $J=$ $5.6 \mathrm{~Hz}, 2 \mathrm{H}), 7.88$ (d, $J=15.4 \mathrm{~Hz}, 1 \mathrm{H}), 7.71-7.39$ (m, 8H), 6.62 (q, $J=8.2 \mathrm{~Hz}, 1 \mathrm{H}) ;{ }^{13} \mathrm{C} \mathrm{NMR}$ (75.5 MHz, $\left.\mathrm{CDCl}_{3}\right) \delta 193.8,190.0,149.2,141.0,138.1,135.6,134.4,133.6,131.9,131.4$, 130.8, 129.2, 129.0, 128.3, 128.1, 126.3; HRMS (CI, isobutane) calcd for $\mathrm{MH}^{+}$263.107205, found 263.107547.

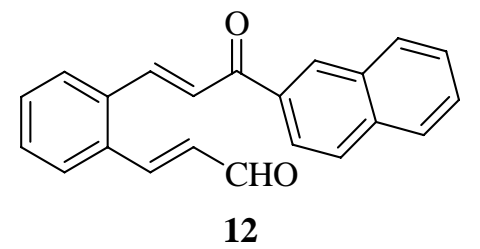

${ }^{1} \mathrm{H}$ NMR (400 MHz, $\left.\mathrm{CDCl}_{3}\right) \delta 9.78(\mathrm{~d}, J=7.6 \mathrm{~Hz}, 1 \mathrm{H}), 8.55$ (s, 1H), 8.26 (d, $J=15.4 \mathrm{~Hz}, 1 \mathrm{H}), 8.12$ (d, $J=8.6 \mathrm{~Hz}, 1 \mathrm{H}), 8.01-$ 7.90 (m, 4H), 7.81 (d, $J=7.6 \mathrm{~Hz}, 1 \mathrm{H}), 7.69-7.47$ (m, 6H), 6.70 (dd, $J=15.8,7.6 \mathrm{~Hz}, 1 \mathrm{H}) ;{ }^{13} \mathrm{C} \mathrm{NMR}\left(100 \mathrm{MHz}, \mathrm{CDCl}_{3}\right) \delta 193.3$, 189.4, 148.8, 140.6, 135.7, 135.3, 135.2, 134.1, 132.6, 131.5, 131.0, 130.4, 130.2, 129.6, 128.8, 128.7, 128.0, 127.9, 127.8, 127.0, 126.1, 124.4; HRMS (EI) calcd for $\mathrm{MH}^{+} 312.115030$, found 312.115074 .<smiles>O=C/C=C/c1ccccc1/C=C/C(=O)c1ccc(Br)s1</smiles>

14

${ }^{1} \mathrm{H}$ NMR (300 MHz, $\left.\mathrm{CDCl}_{3}\right) \delta 9.70(\mathrm{~d}, J=7.6 \mathrm{~Hz}, 1 \mathrm{H}), 8.13$ (d, $J=$ $15.3 \mathrm{~Hz}, 1 \mathrm{H}), 7.85$ (d, $J=15.8 \mathrm{~Hz}, 1 \mathrm{H}), 7.68-7.39$ (m, 5H), 7.217.09 (m, 2H), 6.61 (dd, $J=15.8,7.6 \mathrm{~Hz}, 1 \mathrm{H}) ;{ }^{13} \mathrm{C}$ NMR $(75.5 \mathrm{MHz}$, $\left.\mathrm{CDCl}_{3}\right) \delta 193.7,180.6,149.0,147.1,140.9,135.1,134.5,132.6$, 132.0, 131.9, 131.4, 131.0, 128.3, 128.2, 124.9, 124.0; HRMS (EI) calcd for $\mathrm{MH}^{+} 345.966328$, found 345.966600 .

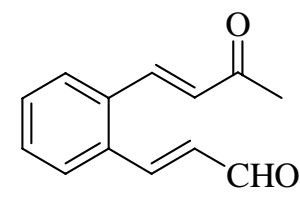

16

${ }^{1} \mathrm{H}$ NMR (300 MHz, $\mathrm{CDCl}_{3}$ ) $\delta 9.78$ (d, $J=7.6,1 \mathrm{H}$ ), 7.89 (dd, $J=10.6$, 15.8Hz, 2H), 7.63 (m, 2H), 7.46 (m, 2H), 6.66 (dd, $J=15.8,8.3 \mathrm{~Hz}, 2 \mathrm{H}$ ), 2.42 (s, 3H); ${ }^{13} \mathrm{C}$ NMR (75.5 MHz, $\left.\mathrm{CDCl}_{3}\right) \delta$ 197.5, 193.3, 148.6, 139.1, 134.6, 133.8, 131.6, 131.0, 130.5, 130.4, 127.9, 127.8, 28.4; HRMS (EI) calcd for $\mathrm{MH}^{+}$200.083730, found 200.083669.<smiles>O=C/C=C/c1ccc(F)cc1/C=C/C(=O)c1ccccc1</smiles>

18

${ }^{1} \mathrm{H}$ NMR (300 MHz, $\left.\mathrm{CDCl}_{3}\right) \delta 9.69$ (d, $\left.J=7.6 \mathrm{~Hz}, 1 \mathrm{H}\right), 8.08$ (d, $J$ $=15.4 \mathrm{~Hz}, 1 \mathrm{H}), 7.99-7.96(\mathrm{~m}, 2 \mathrm{H}), 7.80(\mathrm{~d}, J=15.8 \mathrm{~Hz}, 1 \mathrm{H})$, 7.63-7.53 (m, 2H), 7.49-7.44 (m, 3H), 7.40-7.34 (m, 1H), 7.12 (ddd, $J=8.2,8.2,2.6 \mathrm{~Hz}, 1 \mathrm{H}), 6.57$ (dd, $J=15.8,7.6 \mathrm{~Hz}, 1 \mathrm{H}) ;{ }^{13} \mathrm{C} \mathrm{NMR}$ $\left(75.5 \mathrm{MHz}, \mathrm{CDCl}_{3}\right) \delta 193.6,189.6,166.1,162.7,147.9,139.7,137.9,133.9,131.6,130.6$, 
130.4, 129.3, 129.0, 127.1, 118.3, 118.0, 114.9, 114.6; HRMS (ESI) calcd for $\mathrm{MNa}^{+}$ 305.094828, found 305.094859.<smiles>COc1cc(/C=C/C=O)c(/C=C/C(=O)c2ccccc2)cc1OC</smiles>

20

${ }^{1} \mathrm{H}$ NMR (300 MHz, $\left.\mathrm{CDCl}_{3}\right) \delta 9.67(\mathrm{~d}, J=7.6 \mathrm{~Hz}, 1 \mathrm{H}), 8.14(\mathrm{~d}$, $J=15.4 \mathrm{~Hz}, 1 \mathrm{H}), 7.99-7.95$ (m, 2H), 7.88 (d, $J=15.7 \mathrm{~Hz}, 1 \mathrm{H})$, 7.57-7.43 (m, 3H), 7.33 (d, $J=15.4 \mathrm{~Hz}, 1 \mathrm{H}), 7.08$ (d, $J=22.0$ Hz, 1H), 6.56 (dd, $J=15.6,7.6 \mathrm{~Hz}, 1 \mathrm{H}), 3.96$ (s, 3H), 3.90 (s, 3H); ${ }^{13} \mathrm{C}$ NMR (75.5 MHz, $\left.\mathrm{CDCl}_{3}\right) \delta 193.9,190.2$, 152.0, 151.5, 148.6, 140.6, 138.3, 133.5, 129.9, 129.3, 129.1, 128.9, 128.0, 124.4, 109.7, 109.4, 56.6, 56.5; HRMS (ESI) calcd for $\mathrm{MNa}^{+}$419.182895, found 419.182758.

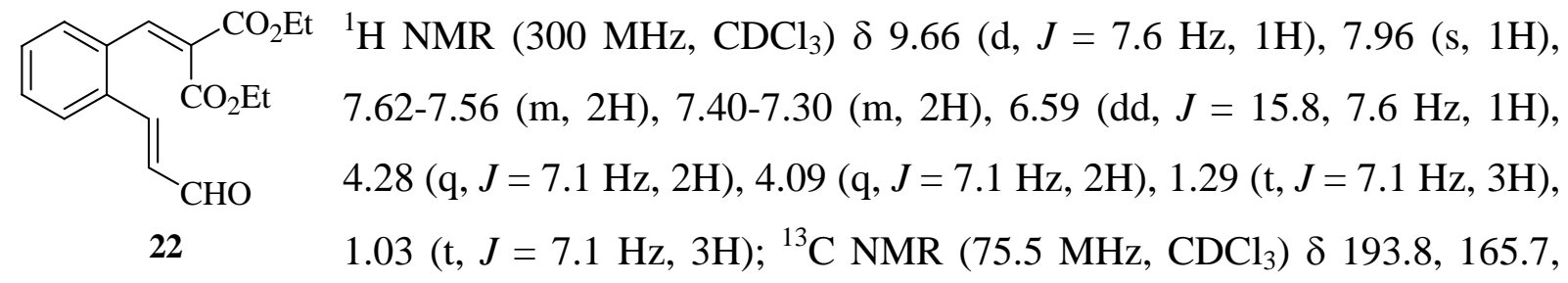
163.8, 149.1, 141.2, 134.5, 133.4, 131.5, 131.2, 131.1, 130.4, 129.3, 127.8, 62.4, 62.0, 14.5, 14.1; HRMS (ESI) calcd for $\mathrm{MNa}^{+} 327.120844$, found 327.120691.

Substrates $\mathbf{2 4}$ and $\mathbf{2 6}$ were prepared according to the procedure of Roush et al. ${ }^{3}$<smiles>O=CC=CC(=O)c1ccccc1</smiles>

24

${ }^{1} \mathrm{H}$ NMR (400 MHz, $\left.\mathrm{CDCl}_{3}\right) \delta 9.46(\mathrm{~d}, J=7.7 \mathrm{~Hz}, 1 \mathrm{H}), 7.85$ (d, $J=7.6 \mathrm{~Hz}$, 2H), 7.51-7.38 (m, 3H), 6.99-6.75 (m, 3H), 6.14-6.08 (m, 1H), 2.51 (m, $4 \mathrm{H}) ;{ }^{13} \mathrm{C}$ NMR (100 MHz, $\left.\mathrm{CDCl}_{3}\right) \delta$ 193.2, 189.9, 155.5, 146.2, 137.3, 133.3, 132.5, 128.2, 128.1, 126.6, 30.7, 30.5; HRMS (CI) calcd for $\mathrm{MH}^{+}$ 215.107206, found 215.106931.<smiles>O=CC=CC(=O)c1ccccc1</smiles>

26

${ }^{1} \mathrm{H}$ NMR (300 MHz, $\left.\mathrm{CDCl}_{3}\right) \delta 9.53(\mathrm{~d}, J=7.9 \mathrm{~Hz}, 1 \mathrm{H}), 7.93$ (d, $J=8.2$ $\mathrm{Hz}, 2 \mathrm{H}), 7.57-7.44$ (m, 3H), 7.09-6.80 (m, 3H), 6.19-6.11 (m, 1H), 2.452.35 (m, 4H), 1.82-1.72 (m, 2H); ${ }^{13} \mathrm{C}$ NMR (75.5 MHz, $\left.\mathrm{CDCl}_{3}\right) \delta$ 193.8, 190.5, 157.3, 148.1, 137.8, 133.5, 132.8, 128.6, 128.5, 126.6, 32.1, 26.3; HRMS (ESI) calcd for $\mathrm{MNa}^{+}$251.104344, found 251.104252.

\footnotetext{
${ }^{3}$ Frank, S. A.; Mergott, D. J.; Roush, W. R. J. Am. Chem. Soc. 2002, 124, 2404 -2405.
} 


\section{Screening of different Hantzsch esters}

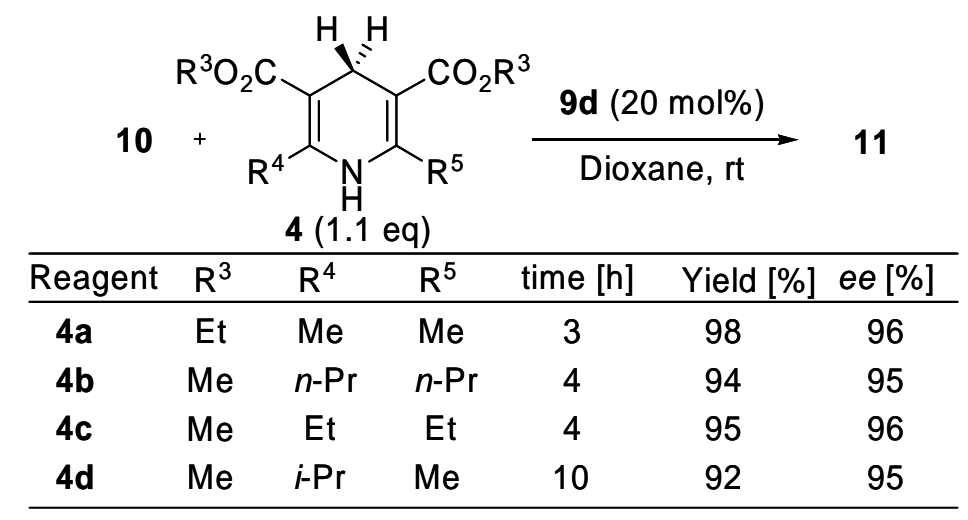

Representative experimental procedure for the catalytic asymmetric reductive Michael cyclization. To a solution of enal enones $10,12,14,16,18,20,22,24$, and 26 (0.5 mmol) and imidazolidinone-based chiral organocatalysts 9a-g (20 mol\%) in dry 1,4-dioxane (5 mL) was added Hantzsch ester 4a (1.1 equiv). The reaction mixture was stirred at room temperature for 2-4 h, after which the solvent was removed and residue was chromatographed on silica gel to give the pure product $(11,13,15,17,19,21,23,25$, and 27$)$.

The synthesis of the racemic compounds were performed according to the above mentioned procedure at room temperature using dibenzylammonium trifluoroacetate (20 mol\%) as the catalyst.<smiles>O=C[C@H]1c2ccccc2C1CC(=O)c1ccccc1</smiles>

11

${ }^{1} \mathrm{H}$ NMR (300 MHz, $\mathrm{CDCl}_{3}$ ) $\delta 9.91$ (d, $\left.J=2.5 \mathrm{~Hz}, 1 \mathrm{H}\right), 7.99-7.96(\mathrm{~m}$, 2H), 7.61-7.55 (m, 1H), 7.50-7.44 (m, 2H), 7.27-7.18 (m, 4H), 4.21-4.14 (m, 1H), 3.55 (dd, $J=17.9,4.7 \mathrm{~Hz}, 1 \mathrm{H}$ ), 3.37-3.25 (m, 2H), 3.15 (dd, $J$ $=16.3,8.9 \mathrm{~Hz}, 1 \mathrm{H}), 3.03-2.95(\mathrm{~m}, 1 \mathrm{H}) ;{ }^{13} \mathrm{C} \mathrm{NMR}\left(75.5 \mathrm{MHz}, \mathrm{CDCl}_{3}\right) \delta$ 202.2, 198.5, 143.8, 141.1, 136.6, 133.4, 128.7, 128.1, 127.5, 127.1, 124.8, 123.9, 57.5, 44.3, 41.2, 31.8; HRMS (EI) calcd for $\mathrm{MH}^{+}$264.115030, found 264.115096; The enantiomeric excess was determined to be $96 \%$ by chiral HPLC (ChiralPak AD-H column, 5\% i$\mathrm{PrOH} /$ heptane, $1.0 \mathrm{~mL} / \mathrm{min}, 220 \mathrm{~nm}, \mathrm{t}_{\mathrm{R}}$ (minor $16.2 \mathrm{~min}$ ), $\mathrm{t}_{\mathrm{R}}$ (major $17.6 \mathrm{~min}$ ).

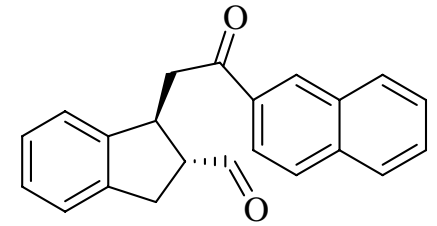

13

${ }^{1} \mathrm{H}$ NMR (300 MHz, $\left.\mathrm{CDCl}_{3}\right) \delta 9.85$ (d, $\left.J=1.7 \mathrm{~Hz}, 1 \mathrm{H}\right), 8.38$ (s, 1H), 7.95 (dd, $J=8.6,1.7 \mathrm{~Hz}, 1 \mathrm{H}), 7.85-7.76$ (m, 3H), 7.53-7.42 (m, 2H), 7.18-7.10 (m, 4H), 4.17-4.10 (m, 1H), 3.58 (dt, $J=17.8$, $4.8 \mathrm{~Hz}, 1 \mathrm{H}), 3.38-3.19$ (m, 2H), 3.07 (dd, $J=16.1,8.8 \mathrm{~Hz}, 1 \mathrm{H}$ ), 2.97-2.90 (m, 1H); ${ }^{13} \mathrm{C}$ NMR (75.5 MHz, $\left.\mathrm{CDCl}_{3}\right) \delta$ 202.6, 198.9, 144.3, 141.6, 136.1, 134.4, 132.9, 130.3, 130.0, 129.1, 128.2, 127.9, 127.5, 127.3, 125.3, 124.4, 124.1, 58.0, 44.8, 41.8, 
32.2; HRMS (EI) calcd for $\mathrm{MH}^{+}$312.115030, found 312.115074; The enantiomeric excess was determined to be $94 \%$ by chiral HPLC (ChiralPak AS-H column, 15\% $\mathrm{i}$-PrOH/heptane, 1.0 $\mathrm{mL} / \mathrm{min}, 220 \mathrm{~nm}, \mathrm{t}_{\mathrm{R}}$ (minor $22.1 \mathrm{~min}$ ), $\mathrm{t}_{\mathrm{R}}$ (major $35.2 \mathrm{~min}$ ).

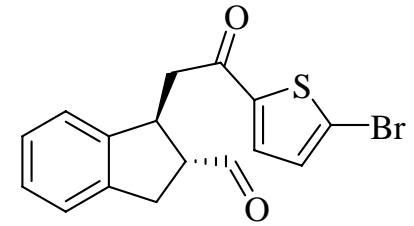

15

${ }^{1} \mathrm{H}$ NMR $\left(300 \mathrm{MHz}, \mathrm{CDCl}_{3}\right) \delta 9.78(\mathrm{~d}, J=2.2 \mathrm{~Hz}, 1 \mathrm{H}), 7.37$ (d, $J=$ 4.1 Hz, 1H), 7.20-7.11 (m, 4H), 7.03 (d, $J=4.1 \mathrm{~Hz}, 1 \mathrm{H}), 4.10-4.03$ (m, 1H), 3.31 (dd, $J=17.1,5.1 \mathrm{~Hz}, 1 \mathrm{H}$ ), 3.23 (dd, $J=16.2,6.3 \mathrm{~Hz}$, 1H), 3.11 (dd, $J=8.7,4.6 \mathrm{~Hz}, 1 \mathrm{H}$ ), 3.06 (dd, $J=8.7,5.6 \mathrm{~Hz}, 1 \mathrm{H}$ ), 2.98-2.91 (m, 1H); ${ }^{13} \mathrm{C}$ NMR (75.5 MHz, $\left.\mathrm{CDCl}_{3}\right) \delta 202.3$, 190.7, 145.7, 143.7, 141.3, 132.6, 131.8, 128.0, 127.5, 125.3, 124.3, 123.6, 57.7, 44.5, 41.6, 32.2; HRMS (ESI) calcd for $\mathrm{MNa}^{+}$ 370.971747, found 370.971422; The enantiomeric excess was determined to be $92 \%$ by chiral HPLC (ChiralPak AD-H column, 5\% i-PrOH/heptane, $1.0 \mathrm{~mL} / \mathrm{min}, 220 \mathrm{~nm}, \mathrm{t}_{\mathrm{R}}$ (minor 20.7 $\min ), t_{R}$ (major $24.9 \mathrm{~min}$ ).

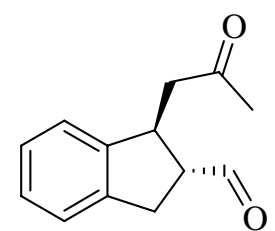

17

${ }^{1} \mathrm{H}$ NMR (300 MHz, $\left.\mathrm{CDCl}_{3}\right) \delta 9.83(\mathrm{t}, J=1.6 \mathrm{~Hz}, 1 \mathrm{H}), 7.26-7.14(\mathrm{~m}, 4 \mathrm{H})$, 4.01-3.94 (m, 1H), 3.26 (dd, $J=16.3,6.8 \mathrm{~Hz}, 1 \mathrm{H}$ ), 3.12 (dd, $J=16.3,8.8 \mathrm{~Hz}$, $1 \mathrm{H}$ ), 3.02 (dd, $J=17.8,5.1 \mathrm{~Hz}, 1 \mathrm{H}$ ), 2.94-2.85 (m, 1H), 2.75 (dd, $J=17.8$, 8.7 Hz, 1H), 2.21 (s, 3H); ${ }^{13} \mathrm{C}$ NMR (75.5 MHz, $\left.\mathrm{CDCl}_{3}\right) \delta$ 207.2, 202.2, 143.6, 140.9, 127.4, 127.1, 124.8, 123.8, 57.6, 49.0, 40.8, 31.9, 30.3; HRMS (EI) calcd for $\mathrm{MH}^{+}$202.099380, found 202.099104; The enantiomeric excess was determined to be $91 \%$ by chiral HPLC (ChiralPak AD-H column, 5\% i-PrOH/heptane, $1.0 \mathrm{~mL} / \mathrm{min}, 220 \mathrm{~nm}, \mathrm{t}_{\mathrm{R}}$ (major $13.5 \mathrm{~min}), \mathrm{t}_{\mathrm{R}}$ (minor $\left.14.4 \mathrm{~min}\right)$.

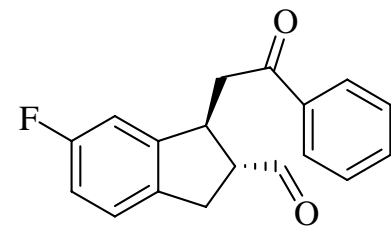

19

${ }^{1} \mathrm{H}$ NMR (300 MHz, $\left.\mathrm{CDCl}_{3}\right) \delta 9.81(\mathrm{~d}, J=2.1 \mathrm{~Hz}, 1 \mathrm{H}), 7.91-7.86(\mathrm{~m}$, 2H), 7.53-7.47 (m, 1H), 7.41-7.35 (m, 2H), 7.11-7.06 (m, 1H), 6.836.76 (m, 2H), 4.09-4.03 (m, 1H), 3.42 (dd, $J=18.0,5.0 \mathrm{~Hz}, 1 \mathrm{H})$, 3.27-3.13 (m, 2H), 3.06-2.90 (m, 2H); ${ }^{13} \mathrm{C} \mathrm{NMR} \mathrm{(75.5} \mathrm{MHz,} \mathrm{CDCl}_{3}$ ) $\delta$ 202.1, 198.6, 164.4, 161.2, 146.5, 136.8, 133.9, 129.2, 128.5, 126.1, 115.0, 114.7, 111.7, 111.4, 58.3, 44.4, 41.5, 31.4; HRMS (ESI) calcd for $\mathrm{MNa}^{+}$305.094828, found 305.094859; The enantiomeric excess was determined to be $97 \%$ by chiral HPLC (ChiralPak AS-H column, 15\% i-PrOH/heptane, $1.0 \mathrm{~mL} / \mathrm{min}, 220 \mathrm{~nm}, \mathrm{t}_{\mathrm{R}}$ (minor $20.8 \mathrm{~min}$ ), $\mathrm{t}_{\mathrm{R}}$ (major $30.2 \mathrm{~min}$ ).

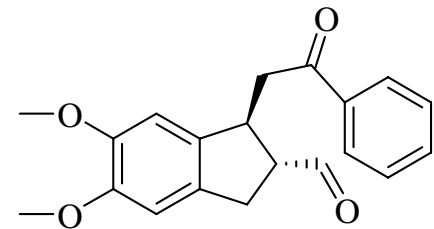

21

${ }^{1} \mathrm{H}$ NMR (300 MHz, $\mathrm{CDCl}_{3}$ ) $\delta 9.81$ (d, $\left.J=2.1 \mathrm{~Hz}, 1 \mathrm{H}\right), 7.90-7.67$ (m, 2H), 7.52-7.46 (m, 1H), 7.41-7.36 (m, 2H), 6.67 (d, $J=8.8 \mathrm{~Hz}$, 2H), 4.06-4.02 (m, 1H), 3.77 (s, 3H), 3.74 (s, 3H), 3.42 (dd, $J=$ 
17.8, 4.9 Hz, 1H), 3.22 (dt, $J=13.4,8.9 \mathrm{~Hz}, 1 \mathrm{H}), 3.15$ (t, $J=5.3 \mathrm{~Hz}, 1 \mathrm{H}), 3.01$ (dd, $J=15.8$, 8.7 Hz, 1H), 2.94-2.87 (m, 1H); ${ }^{13} \mathrm{C}$ NMR (75.5 MHz, $\left.\mathrm{CDCl}_{3}\right) \delta$ 202.7, 199.1, 149.3, 149.0, 137.1, 135.7, 133.8, 133.1, 129.1, 128.4, 108.1, 107.5, 58.1, 56.5, 45.0, 41.9, 31.9; HRMS (ESI) calcd for $\mathrm{MNa}^{+}$347.125381, found 347.125686; The enantiomeric excess was determined to be $96 \%$ by chiral HPLC (ChiralPak AD-H column, 10\% i-PrOH/heptane, 1.0 $\mathrm{mL} / \mathrm{min}, 220 \mathrm{~nm}, \mathrm{t}_{\mathrm{R}}$ (minor $23.4 \mathrm{~min}$ ), $\mathrm{t}_{\mathrm{R}}$ (major $29.7 \mathrm{~min}$ ).

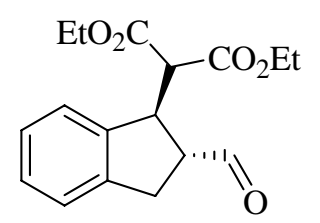

23

${ }^{1} \mathrm{H}$ NMR (300 MHz, $\left.\mathrm{CDCl}_{3}\right) \delta 9.72(\mathrm{~d}, J=1.4 \mathrm{~Hz}, 1 \mathrm{H}), 7.15-7.06(\mathrm{~m}, 4 \mathrm{H})$, 4.23 (dd, $J=6.9,4.2 \mathrm{~Hz}, 1 \mathrm{H}), 4.14$ (q, $J=7.1 \mathrm{~Hz}, 2 \mathrm{H}), 4.02$ (q, $J=7.1 \mathrm{~Hz}$, 2H), 3.72 (d, $J=7.0 \mathrm{~Hz}, 1 \mathrm{H}), 3.40-3.33(\mathrm{~m}, 1 \mathrm{H}), 3.24-3.08(\mathrm{~m}, 2 \mathrm{H}), 1.18$ (t, $J=7.1 \mathrm{~Hz}, 3 \mathrm{H}), 1.04(\mathrm{t}, J=7.1 \mathrm{~Hz}, 3 \mathrm{H}) ;{ }^{13} \mathrm{C} \mathrm{NMR}\left(75.5 \mathrm{MHz}, \mathrm{CDCl}_{3}\right) \delta$ 200.4, 167.3, 167.0, 140.6, 139.6, 126.9, 125.9, 123.8, 123.6, 60.7, 60.5, 54.7, 52.7, 43.6, 30.5, 13.0, 12.8; HRMS (ESI) calcd for $\mathrm{MNa}^{+}$327.120844, found 327.120691; The enantiomeric excess was determined to be $86 \%$ by chiral HPLC (ChiralPak AD-H column, 3\% iPrOH/heptane, $1.0 \mathrm{~mL} / \mathrm{min}, 220 \mathrm{~nm}, \mathrm{t}_{\mathrm{R}}$ (major $13.3 \mathrm{~min}$ ), $\mathrm{t}_{\mathrm{R}}$ (minor $14.9 \mathrm{~min}$ ).

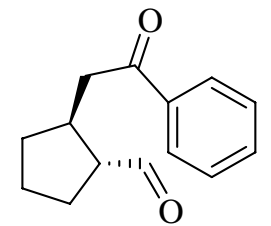

25

All spectroscopic data (HRMS, ${ }^{1} \mathrm{H}$ NMR, ${ }^{13} \mathrm{C}$ NMR) matches data previously reported by List and Shabat et al. ${ }^{4}$ After derivatization of the aldehyde with trimethyl phosphonoacetate (4.0 equiv) and LiOH-mono hydrate (1.5 equiv) proceeds rapidly in THF to form an enone, the enantiomeric excess was determined to be $72 \%$ by chiral HPLC (ChiralPak AD-H column, 5\% i-PrOH/heptane, 1.0 $\mathrm{mL} / \mathrm{min}, 220 \mathrm{~nm}, \mathrm{t}_{\mathrm{R}}$ (major $12.2 \mathrm{~min}$ ), $\mathrm{t}_{\mathrm{R}}$ (minor $15.1 \mathrm{~min}$ ).

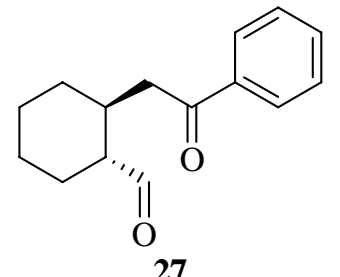

136.0, 132.0, 127.6, 127.1, 54.5, 42.5, 31.6, 30.5, 25.1, 24.3, 23.7 mono hydrate (1.5 equiv) in THF to form an enone, the enantiomeric excess was determined to be $95 \%$ by chiral GC (25 m, IVADEx1/PS086 G376, 0.5 bar $\mathrm{H}_{2}$ as carrier gas): $\mathrm{t}_{\mathrm{R}}$ (minor $154.0 \mathrm{~min}), \mathrm{t}_{\mathrm{R}}$ (major $\left.154.8 \mathrm{~min}\right)$.

\footnotetext{
${ }^{4}$ a) Hechavarria Fonseca, M. T.; List, B. Angew. Chem., Int. Ed. Engl. 2004, 43, 3958-60. b) Weinstain, R.; Lerner, R. A.; Barbas, C. F., III; Shabat, D. J. Am. Chem. Soc. 2005, 127, ASAP.
} 


\section{Determination of the relative configuration}

The trans-configuration of compound $\mathbf{2 5}$ is known. ${ }^{4}$ The relative configuration of aldehyde 27 was deduced from NMR. The trans-configuration of 11, 13, 15, 17, 19, 21, and 23 was assigned analogously. In addition, treating aldehyde 11 with DBU (10 mol\%) in $\mathrm{CH}_{2} \mathrm{Cl}_{2}$ did not result in any measurable isomerization (NMR) as would be expected for the trans-isomer.

\section{Determination of the absolute configuration of 25}

The absolute configuration of $\mathbf{2 5}$ was determined by comparing the optical rotation with the reported value. ${ }^{5}$ In addition, 25 was converted into known ester (-)-(1R,2S) as outlined in Scheme 1.

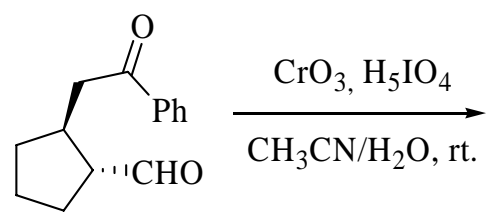

25<smiles>O=C(C[C@@H]1CCC[C@H]1C(=O)O)c1ccccc1</smiles>

$\mathrm{Mg}\left(\mathrm{ClO}_{4}\right)_{2}, \mathrm{CH}_{3} \mathrm{NO}_{2}, \mathrm{rt}$

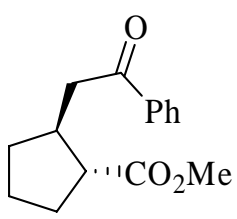

$(-)-(1 R, 2 S)$

Scheme 1. Determination of the absolute stereochemistry of (1R,2S)-2-(2-oxo-2-phenylethyl) -cyclopentanecarboxylic acid methyl ester from 25.

\footnotetext{
${ }^{5}$ a) Enders, D.; Scherer, H. J.; Raabe, G. Angew. Chem., Int. Ed. Engl. 1991, 30, 1664. b) Weinstain, R.; Lerner, R. A.; Barbas, C. F., III; Shabat, D. J. Am. Chem. Soc. 2005, 127, ASAP.
} 
<smiles>O=C[C@@H]1Cc2ccccc2[C@H]1CC(=O)c1ccccc1</smiles>

11<smiles>O=C[C@H]1Cc2ccccc2[C@H]1CC(=O)c1ccc2ccccc2c1</smiles>

13
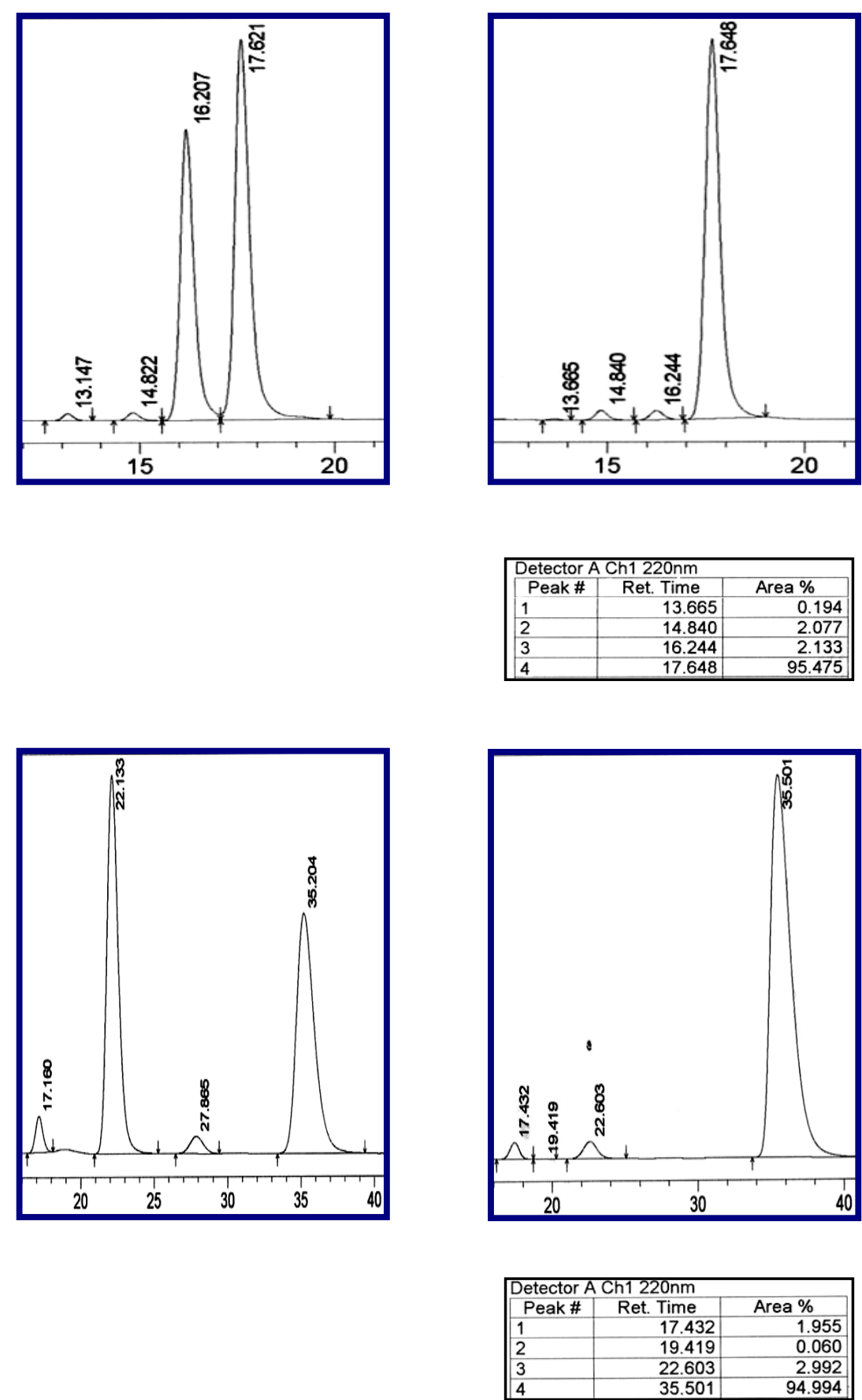
<smiles>O=C[C@@H]1Cc2ccccc2[C@H]1CC(=O)c1ccc(Br)s1</smiles>

15

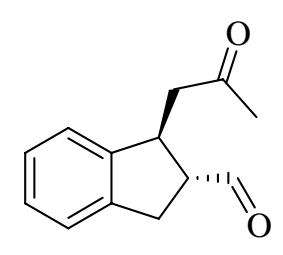

17<smiles>O=C[C@H]1Cc2ccc(F)cc2[C@H]1CC(=O)c1ccccc1</smiles>

19
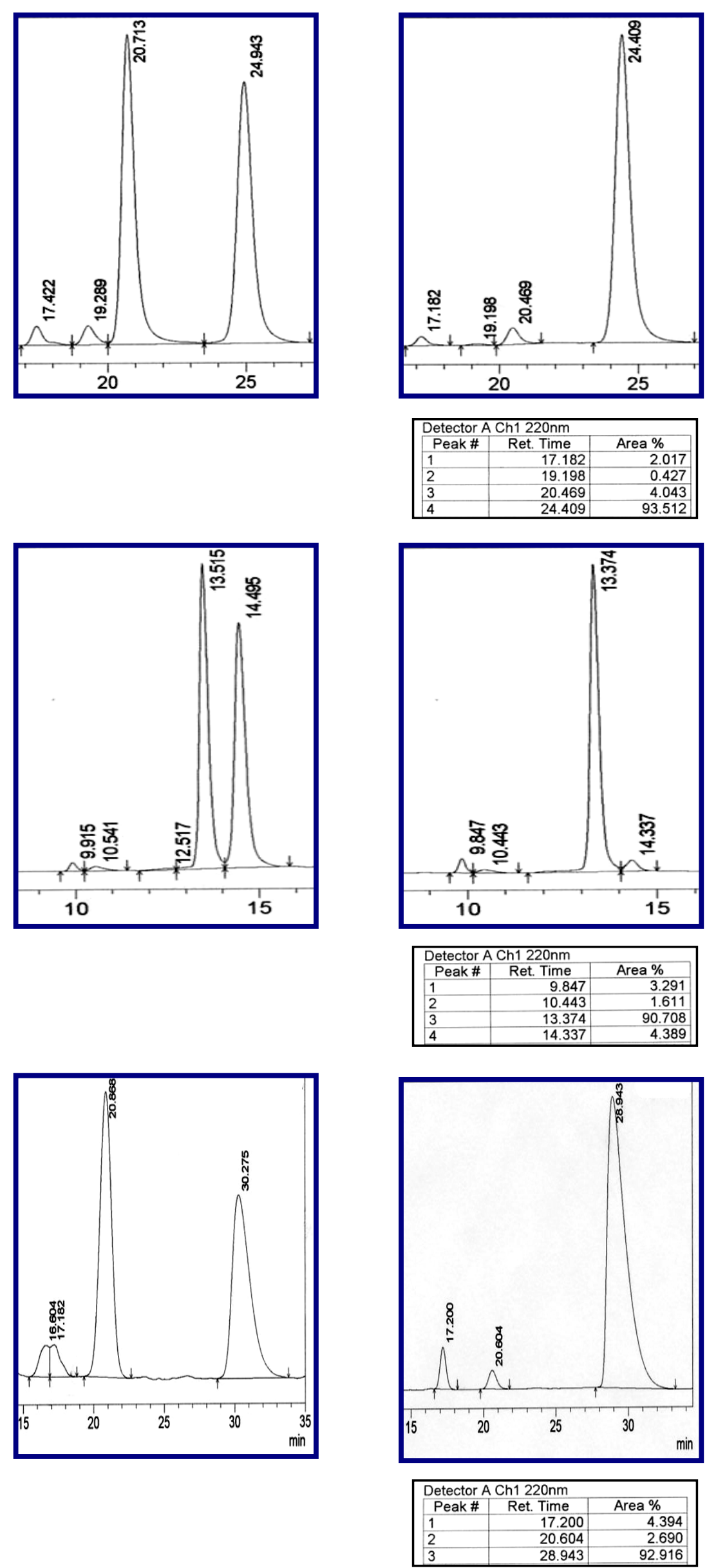

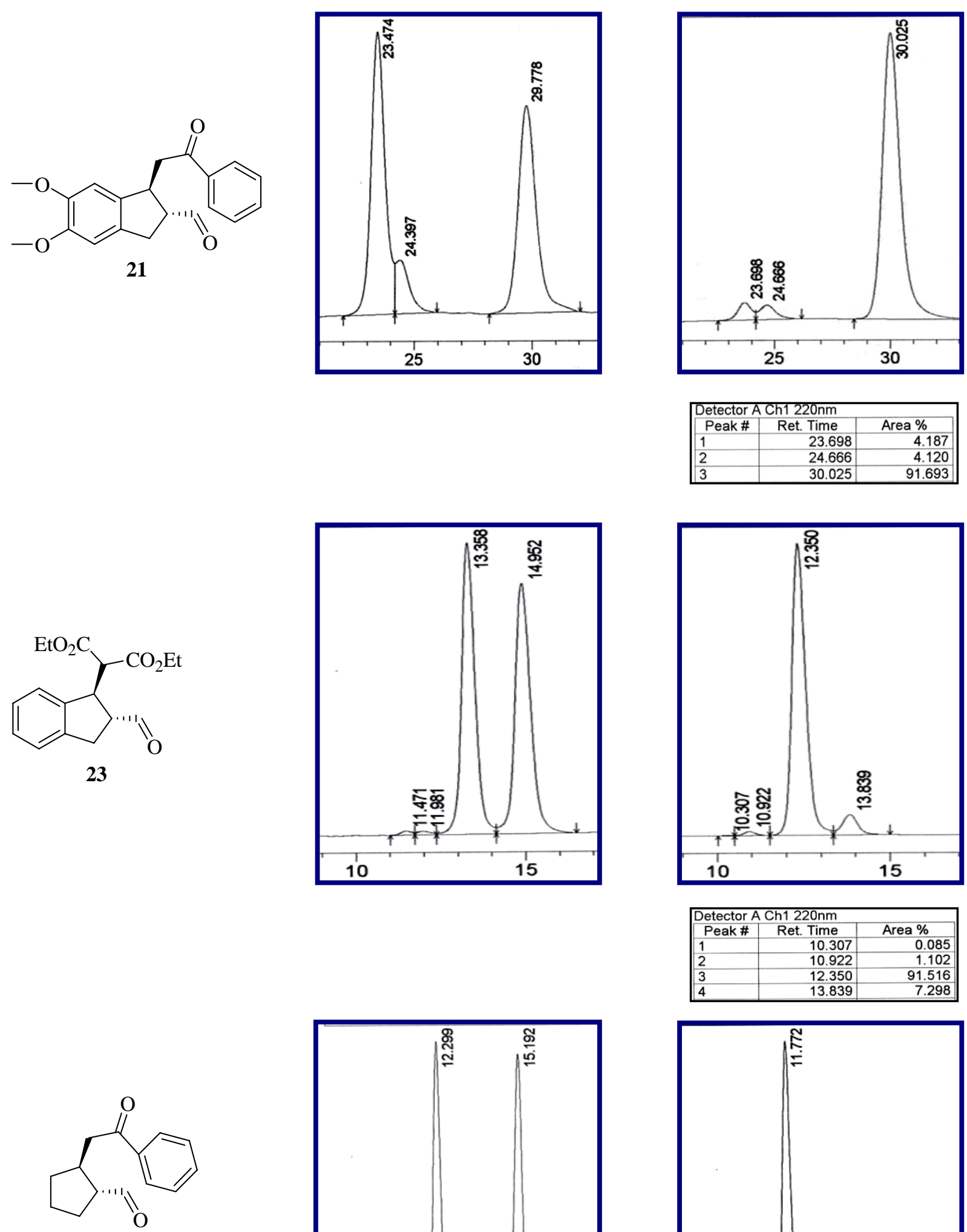

25
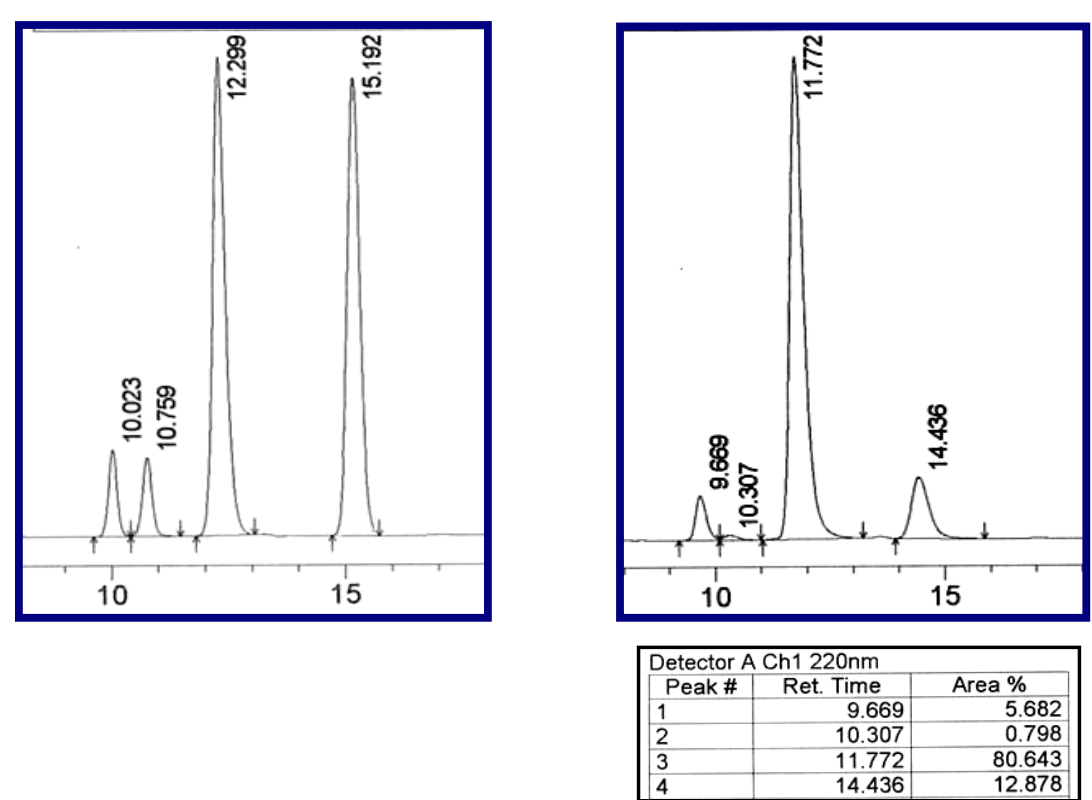

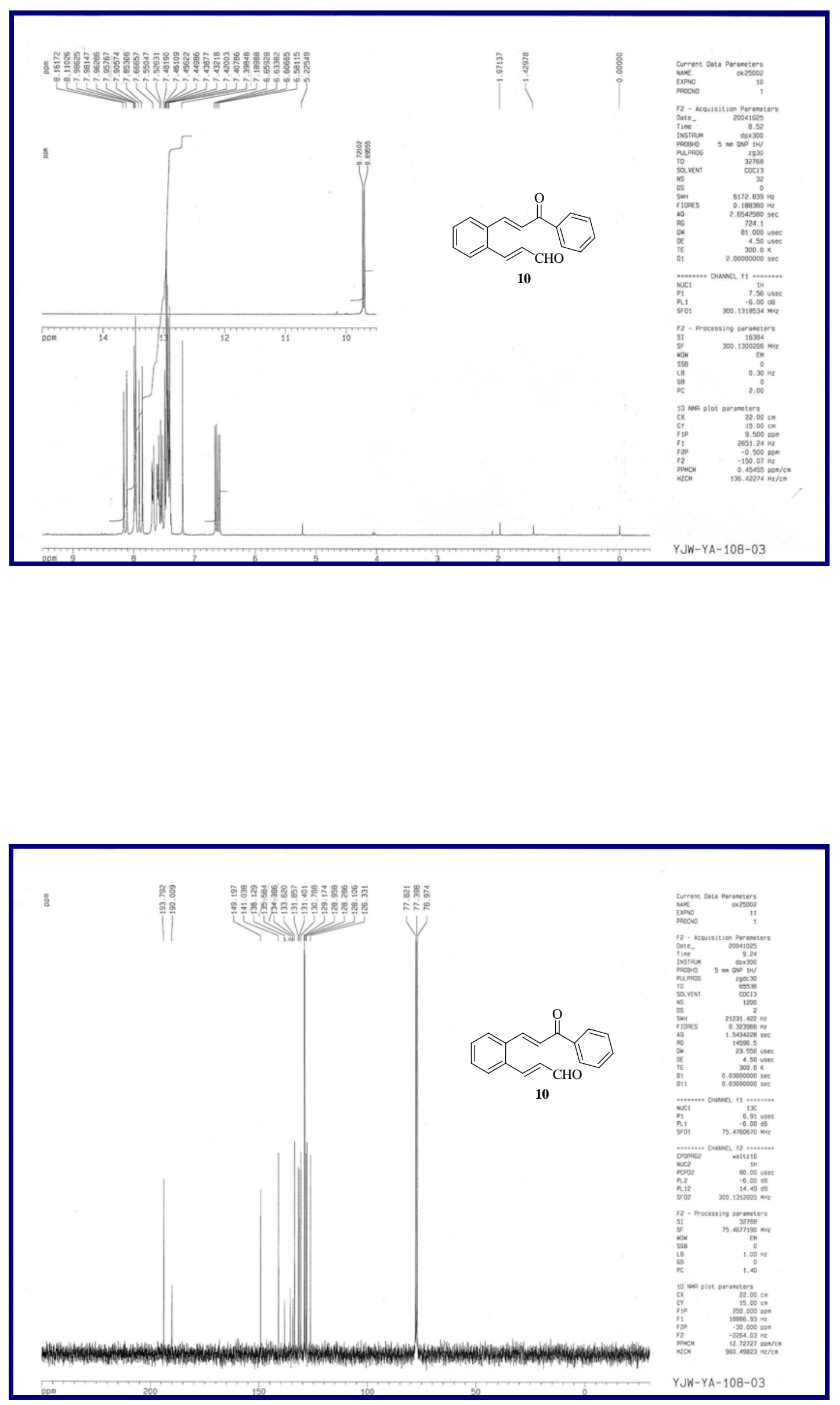

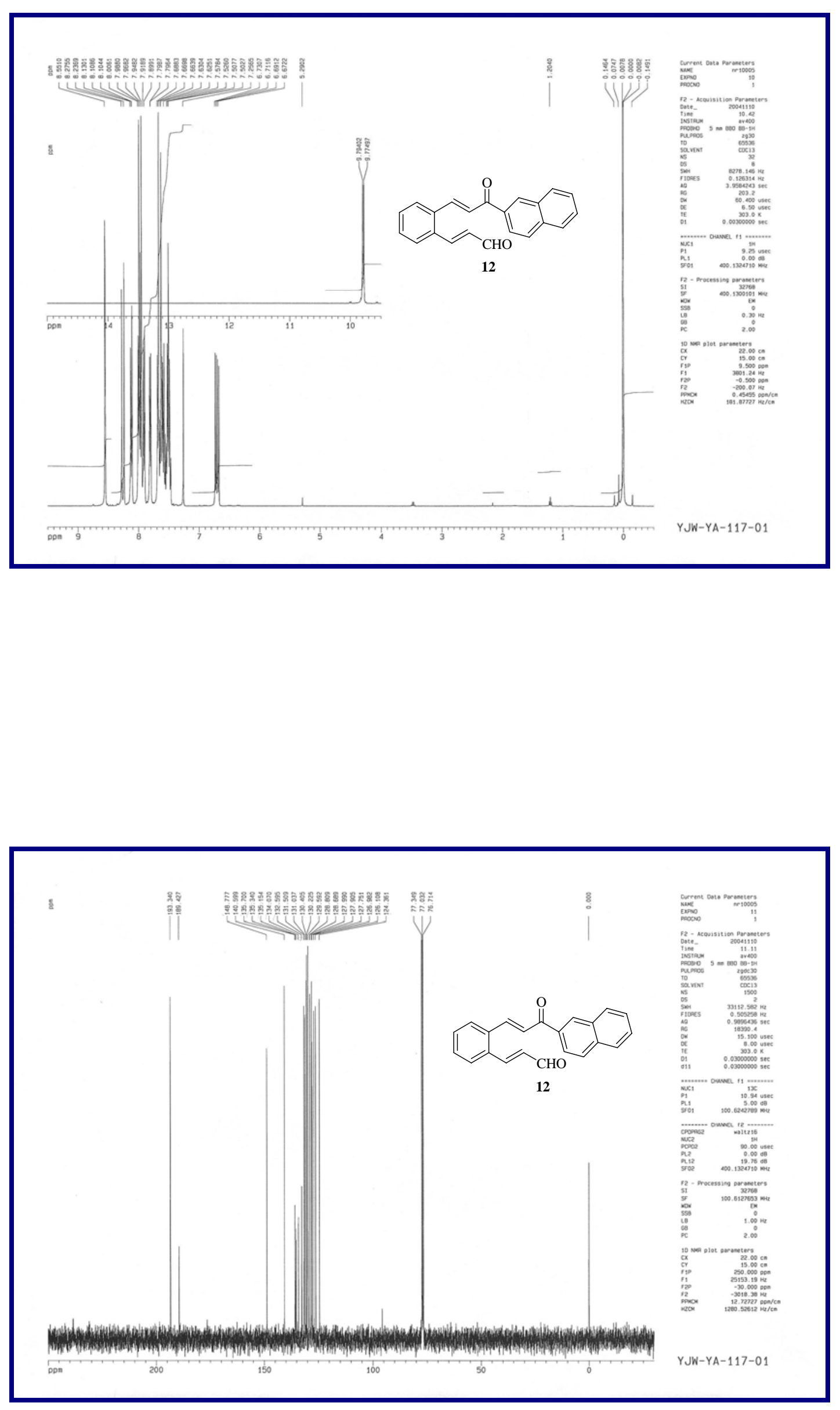

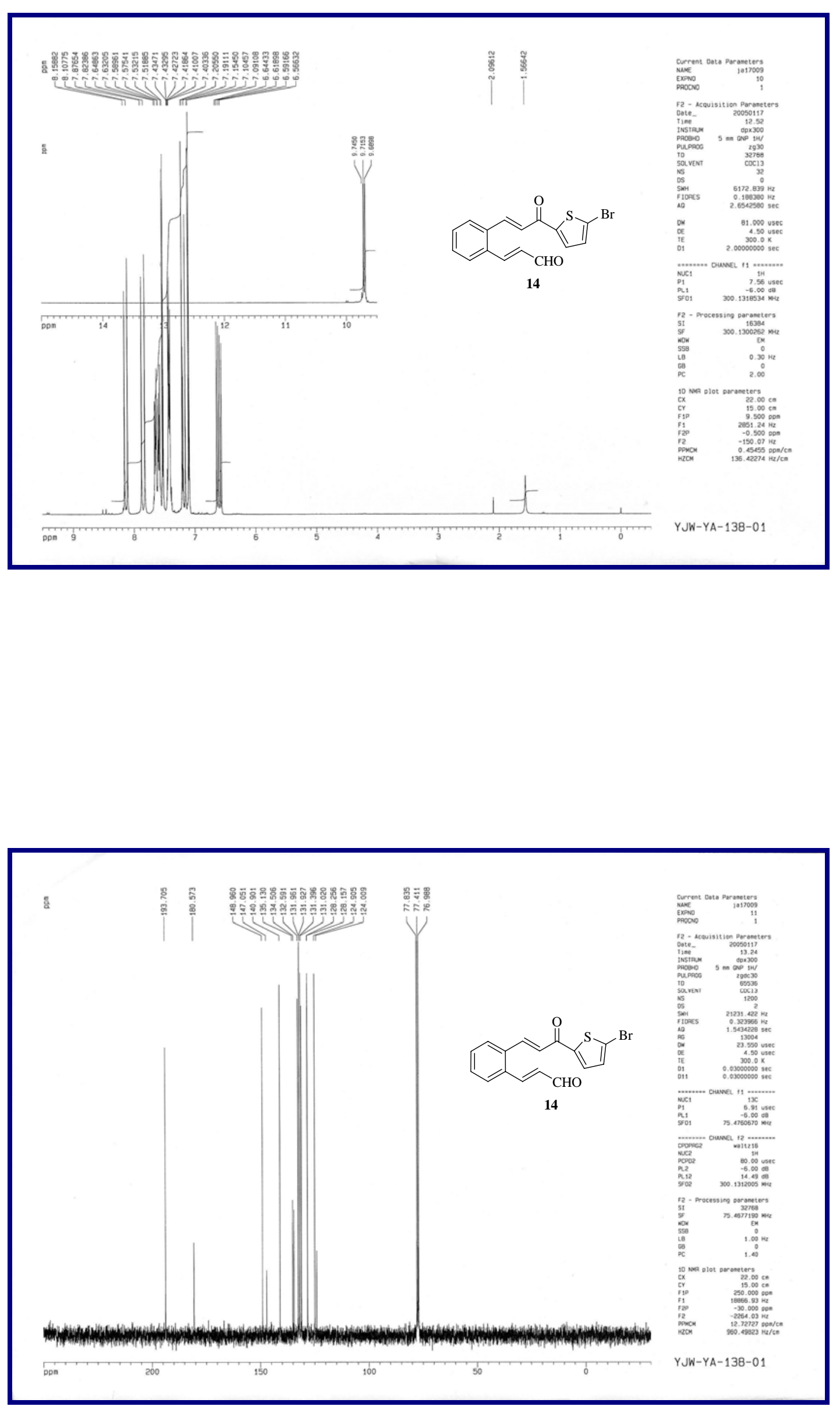

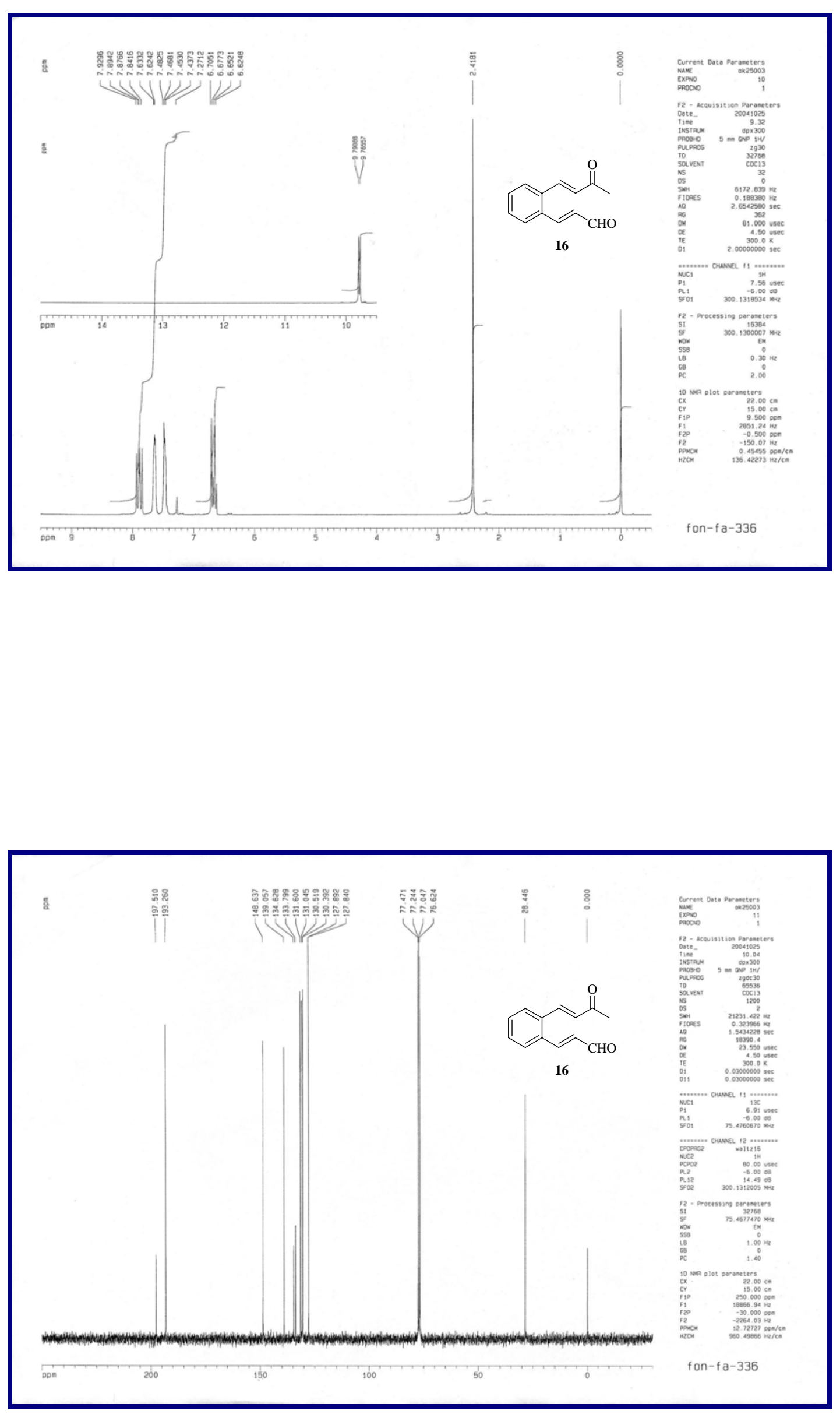

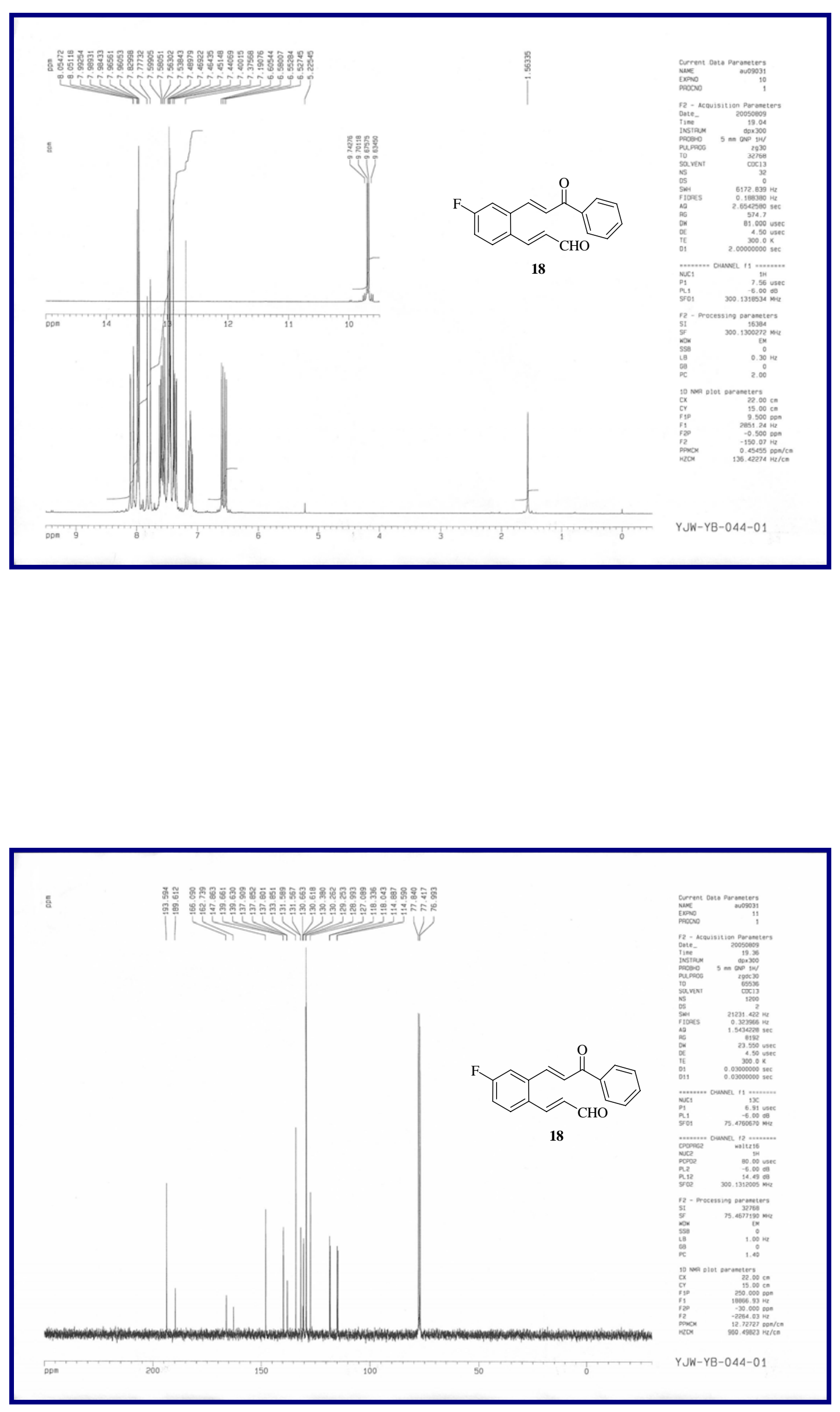

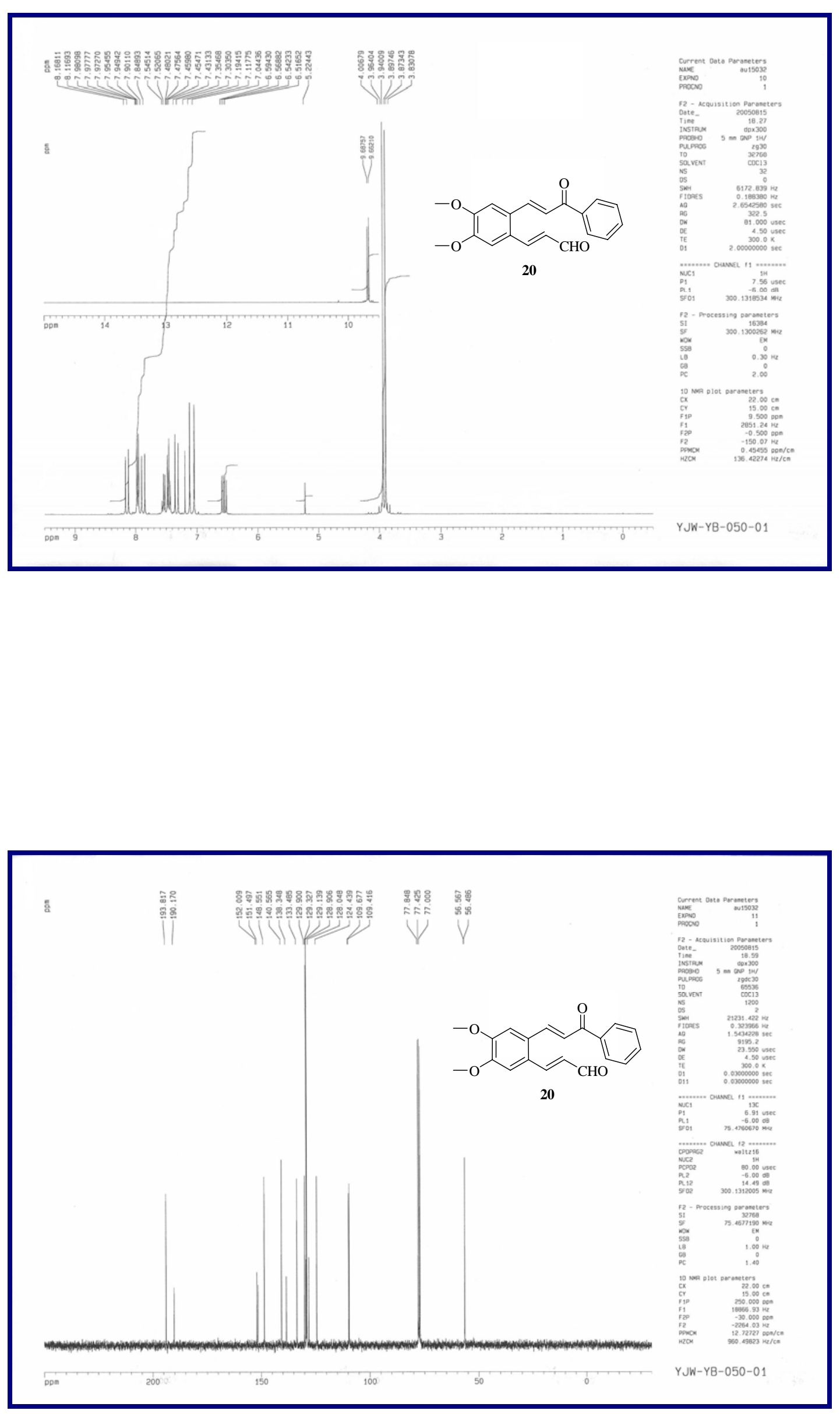

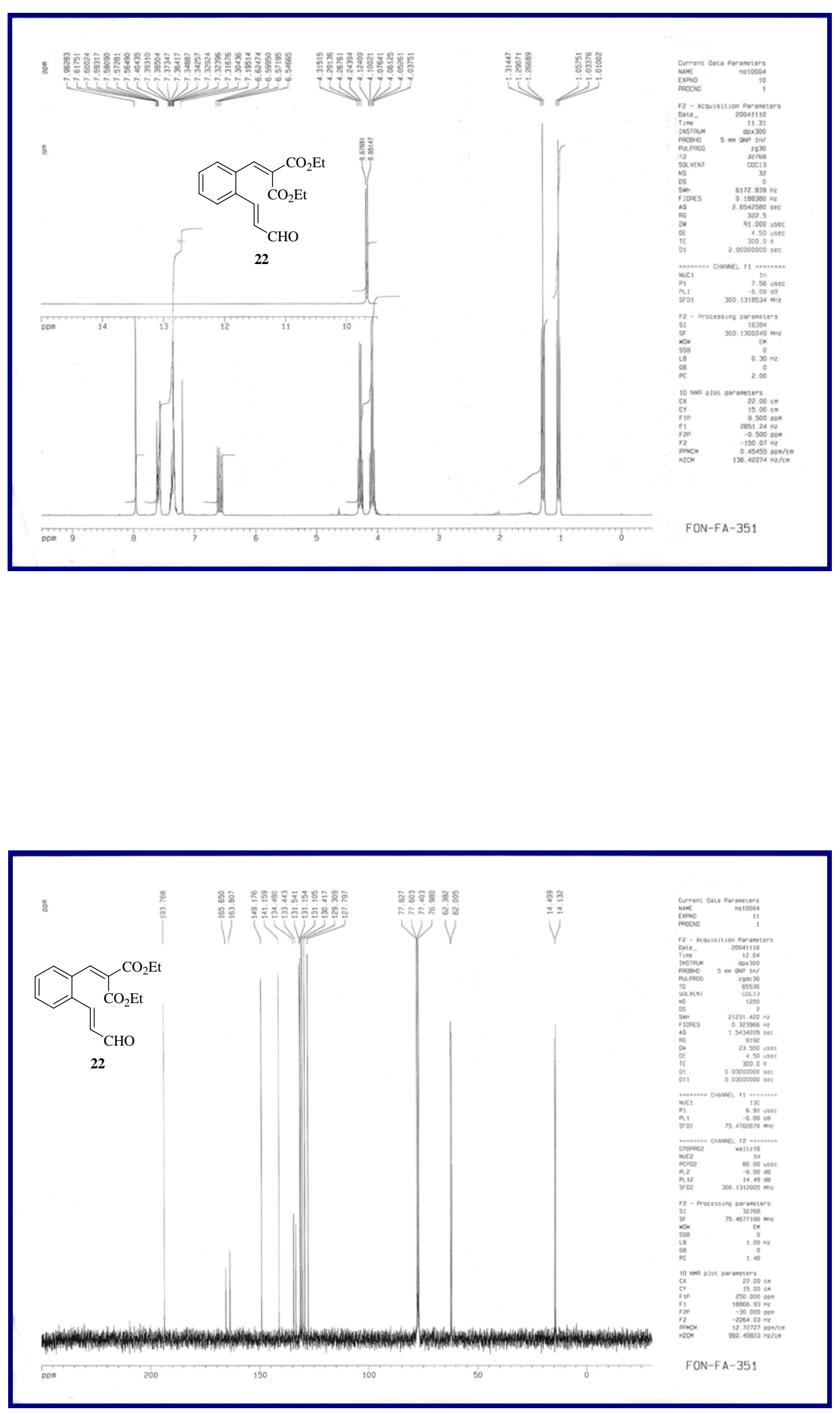

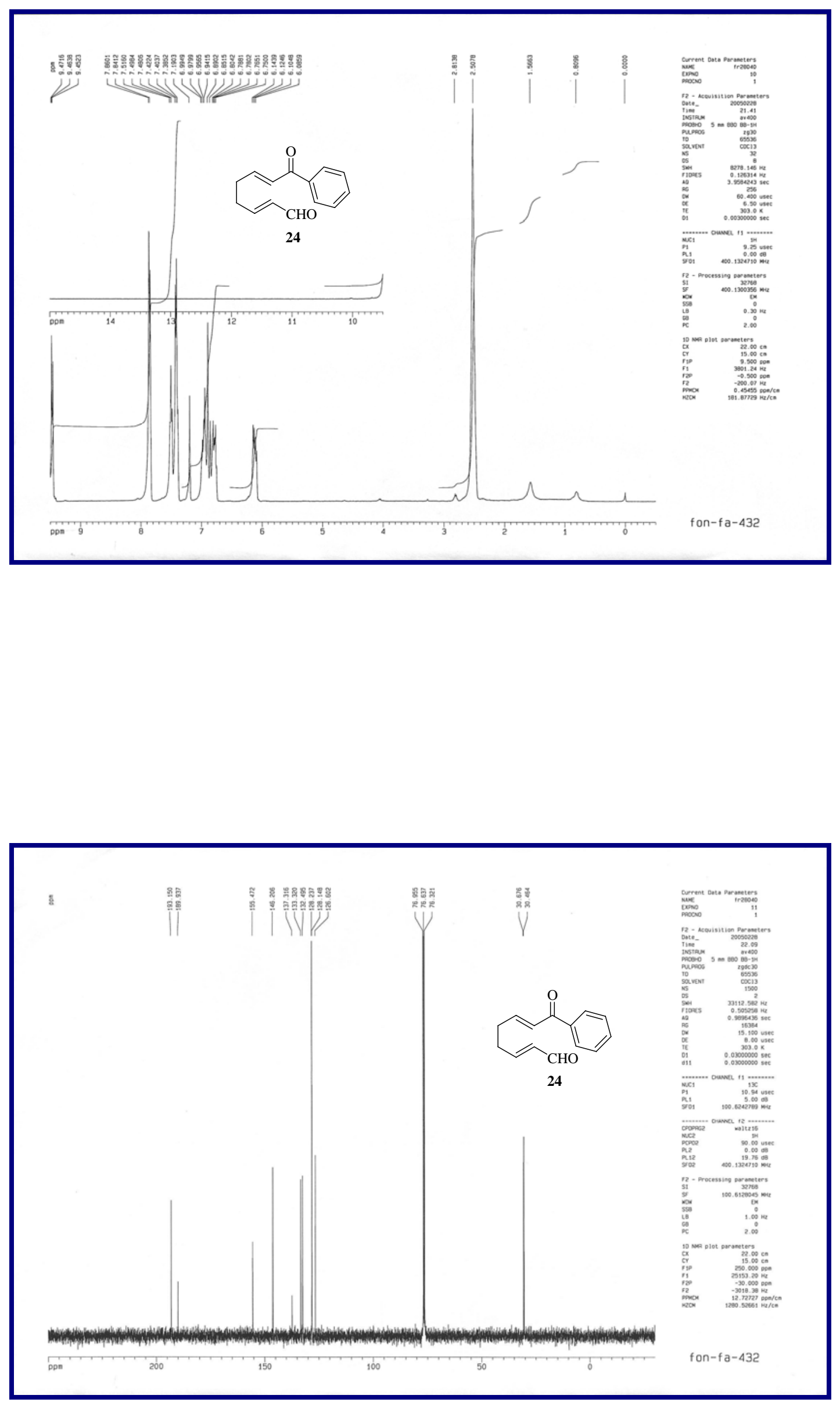

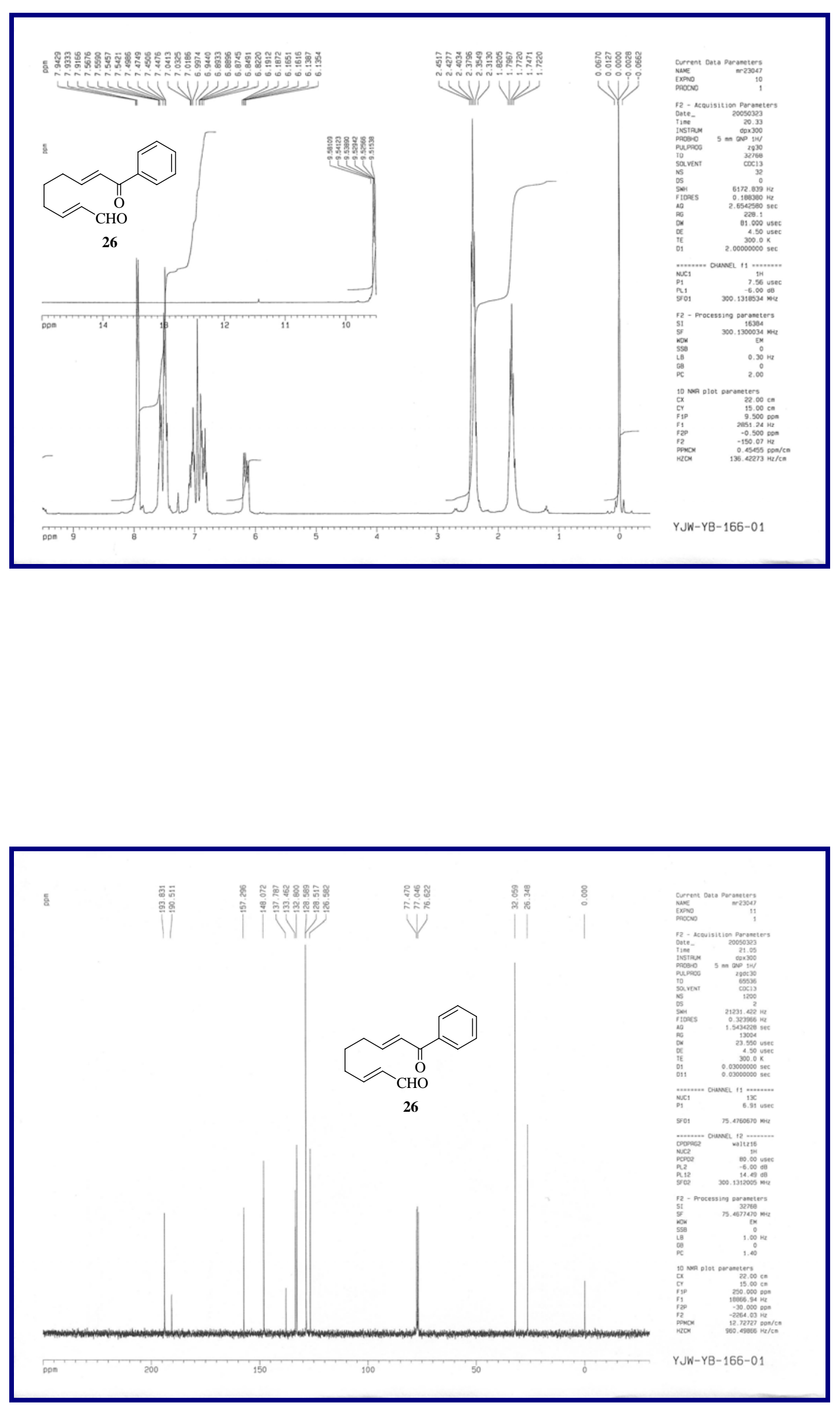

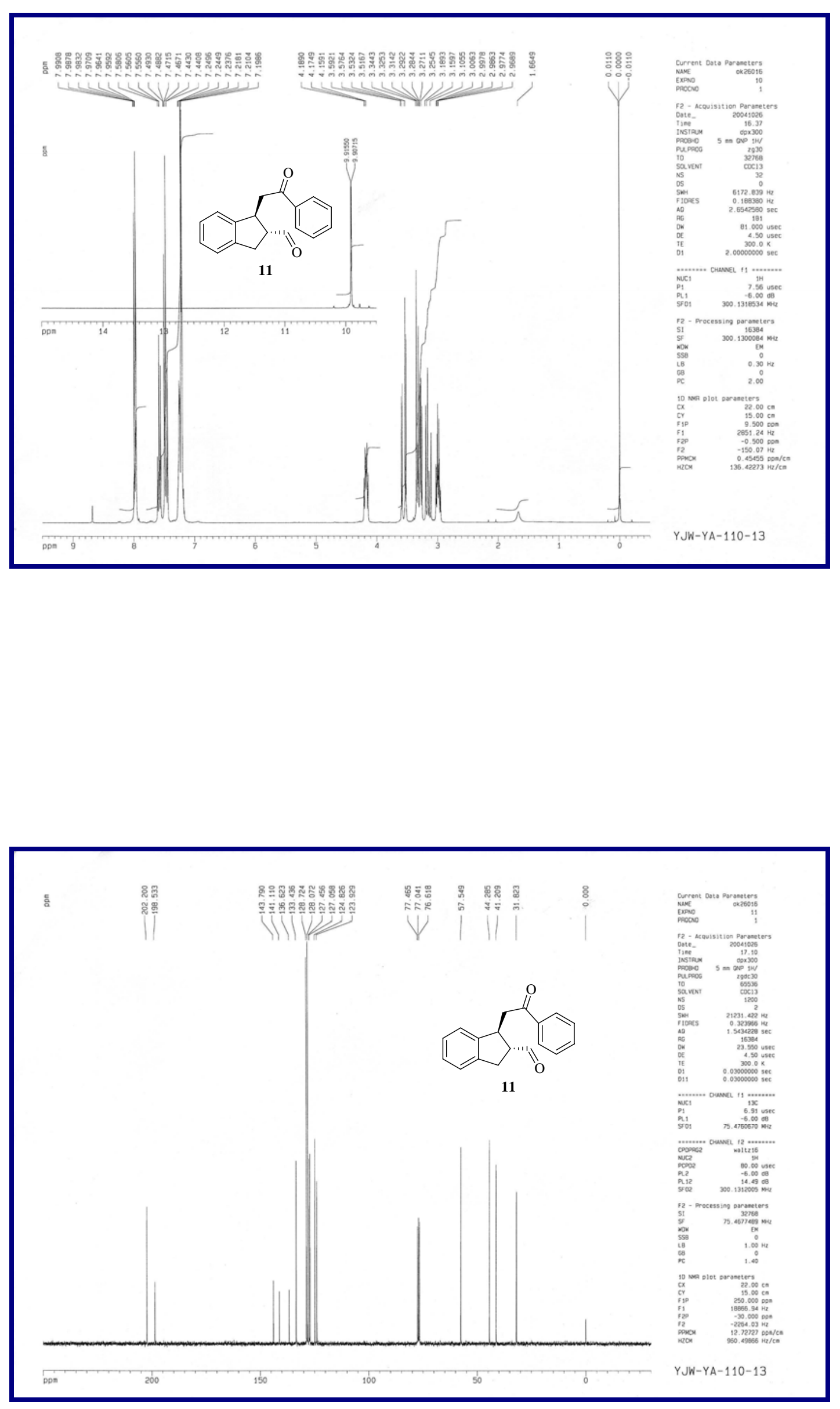

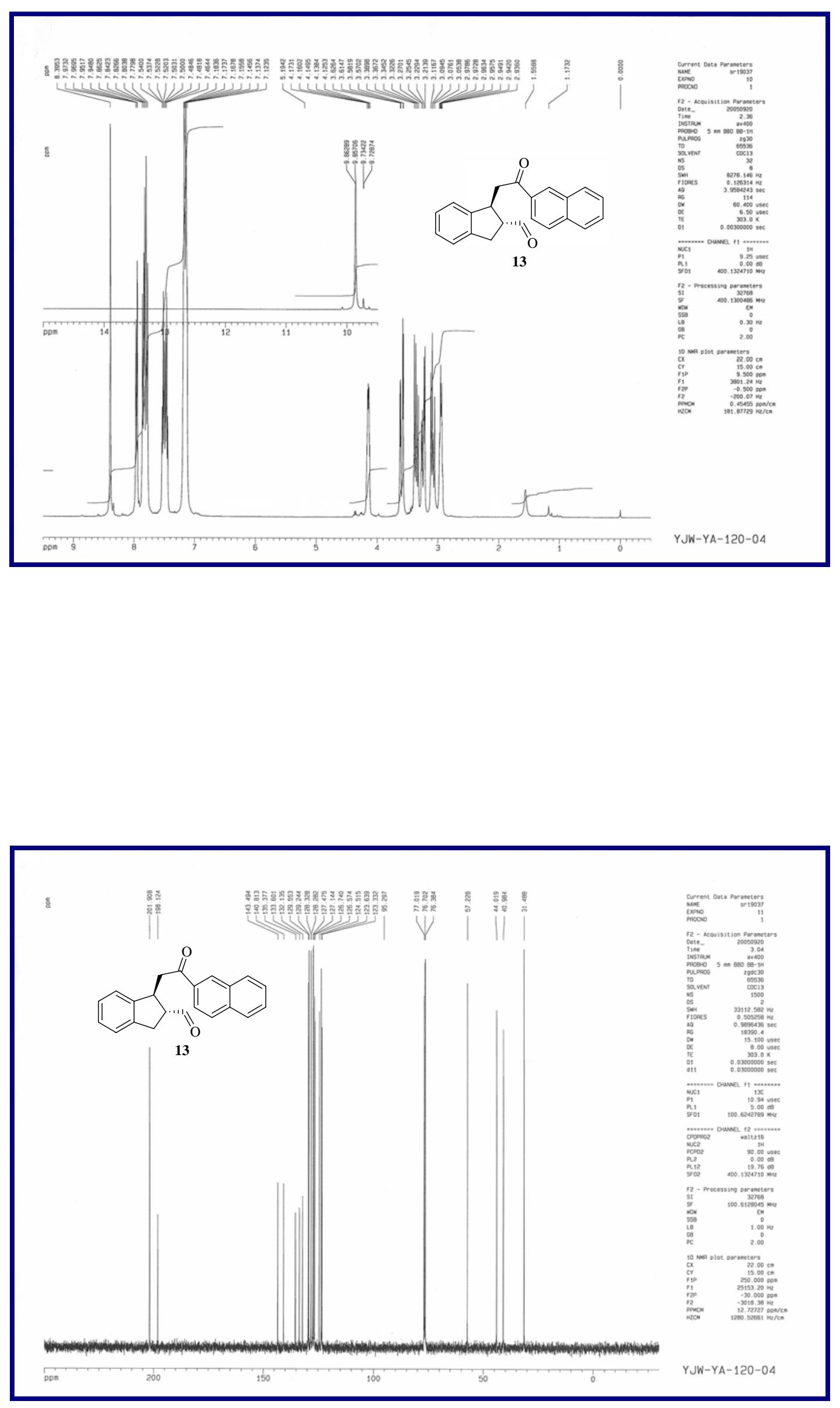

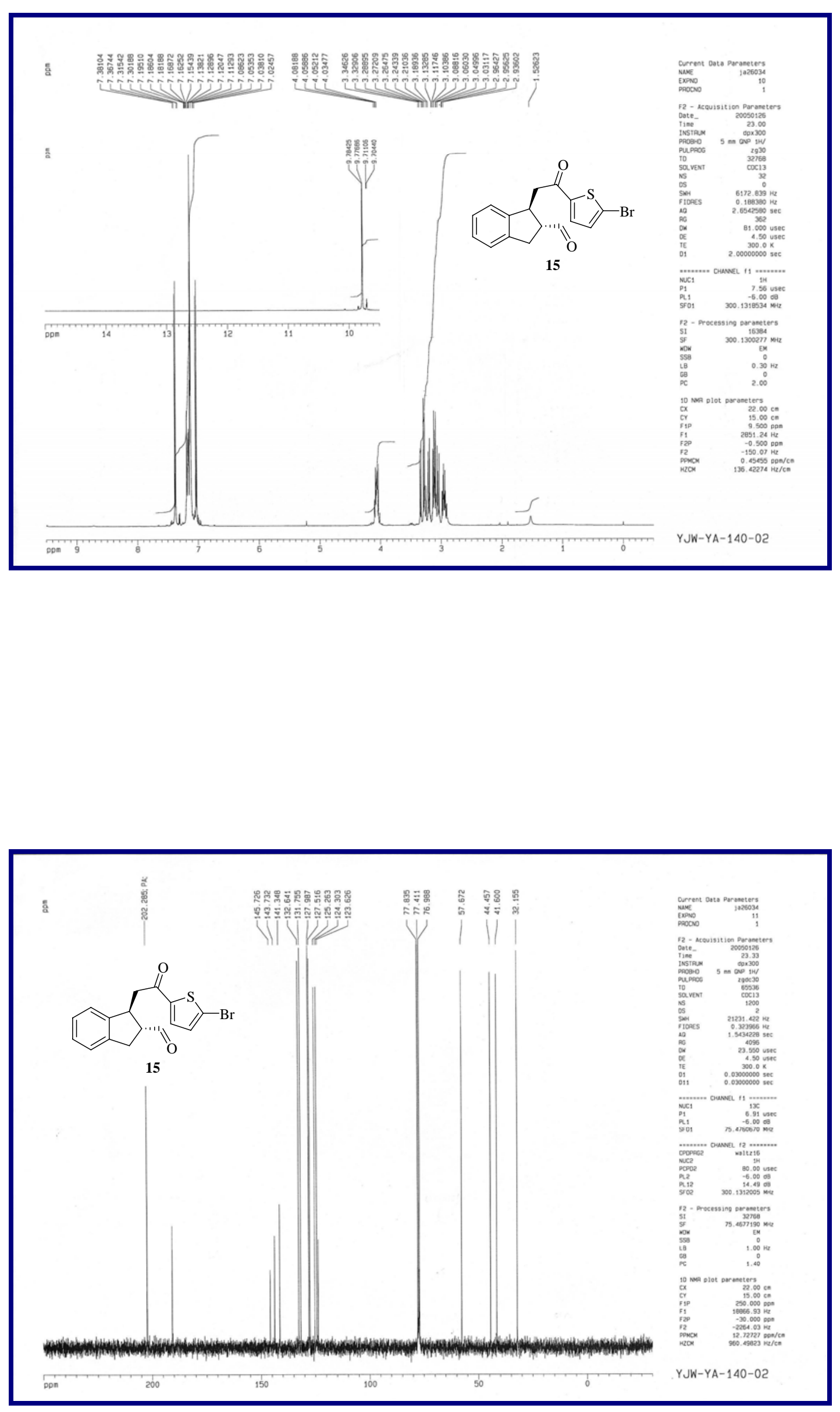

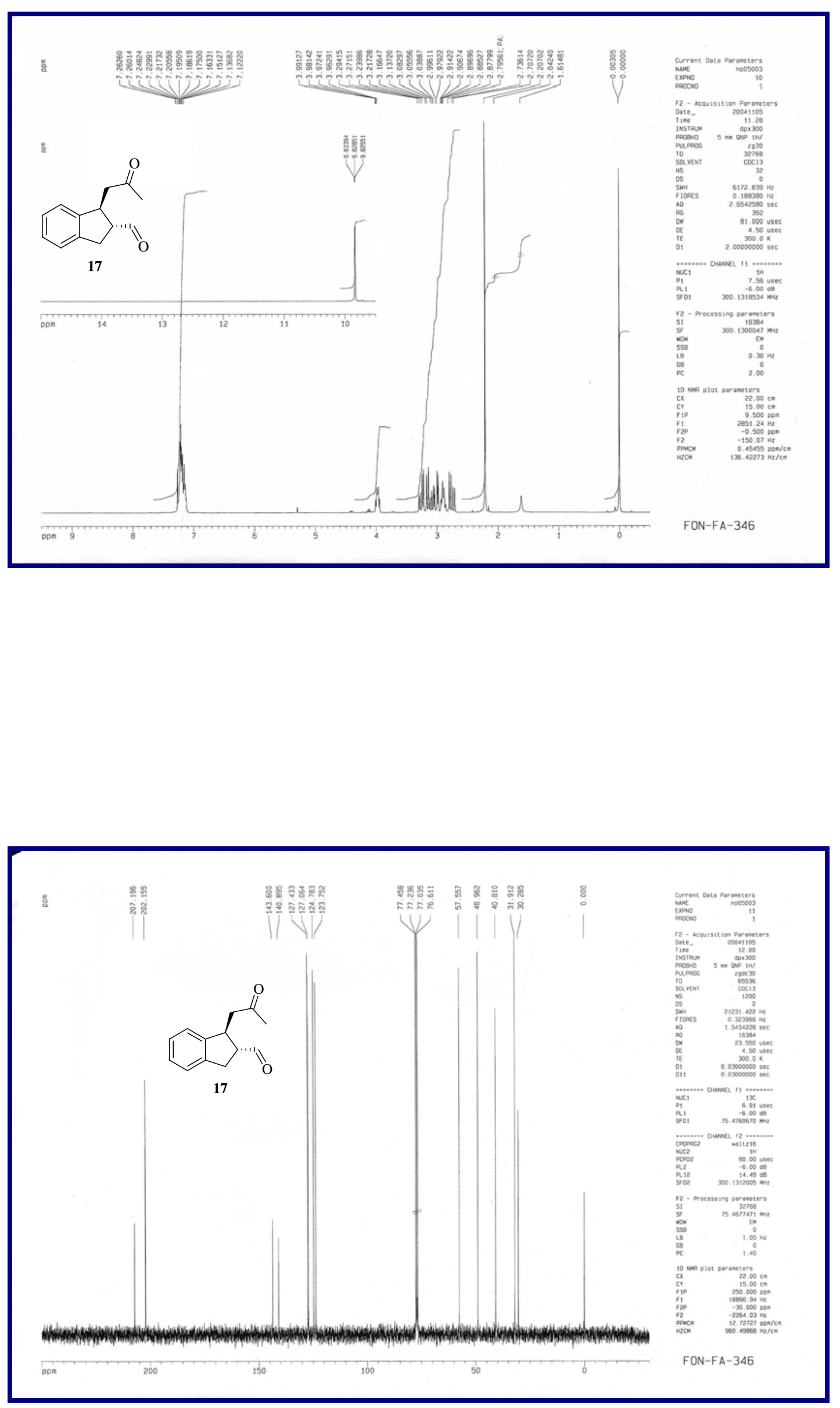

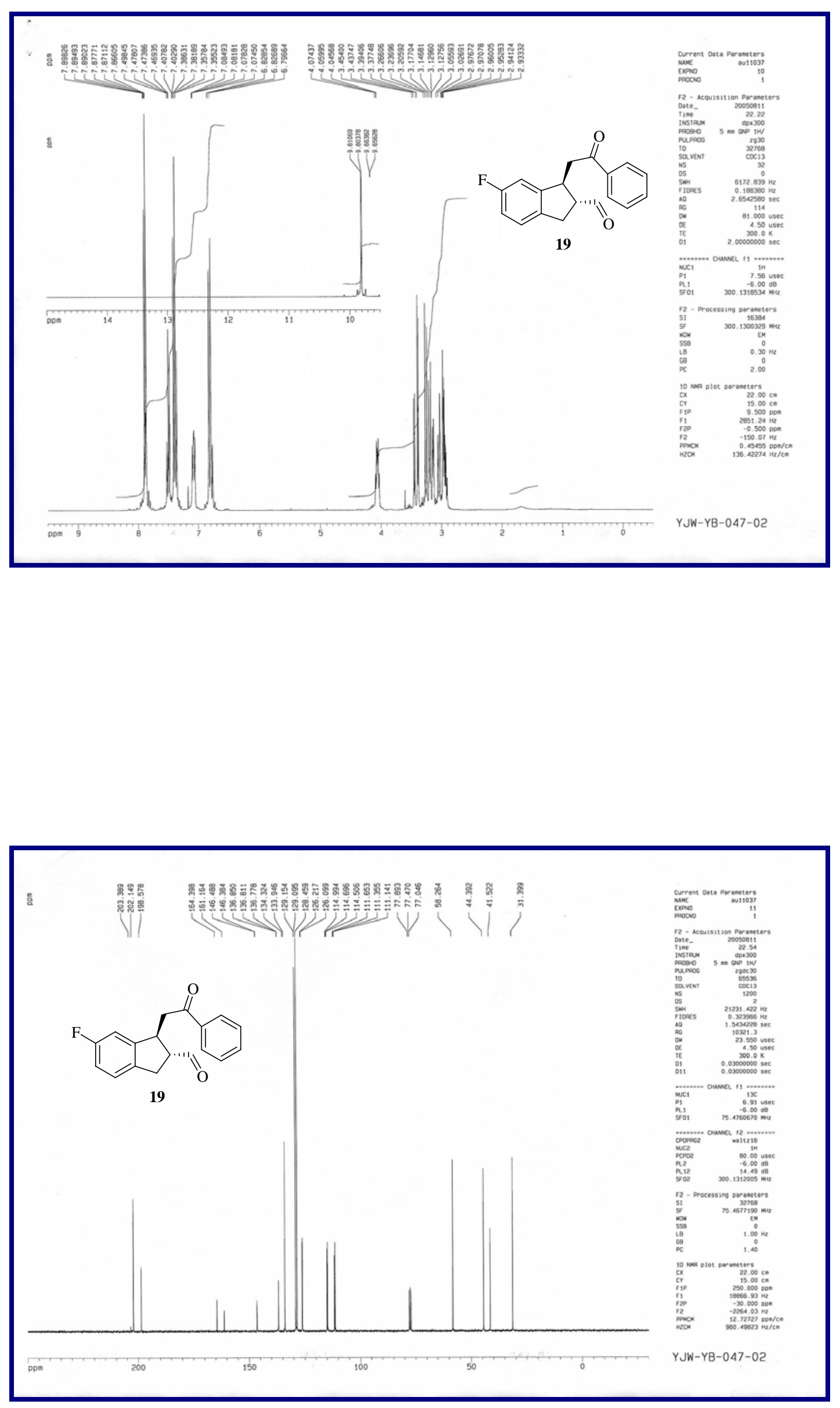

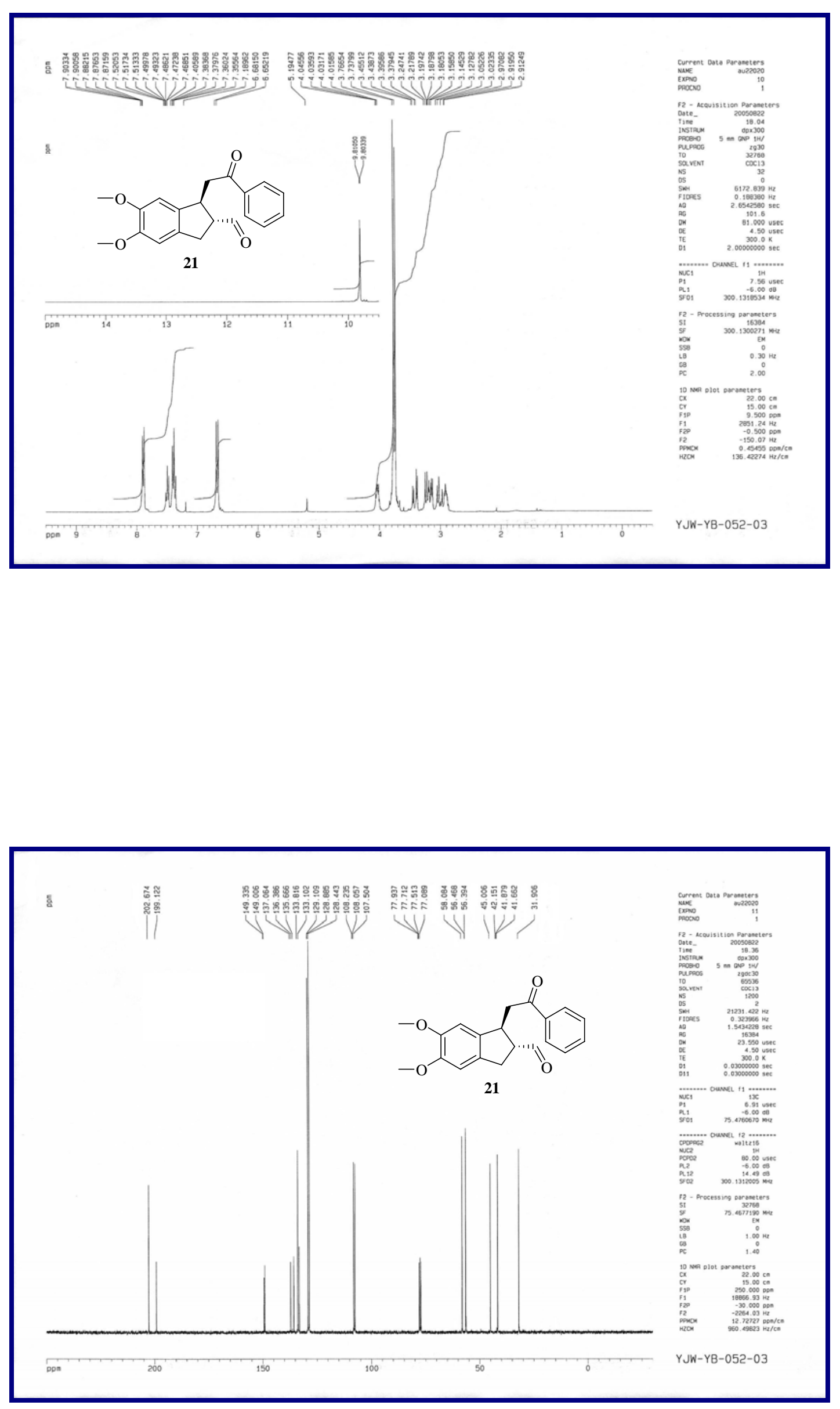

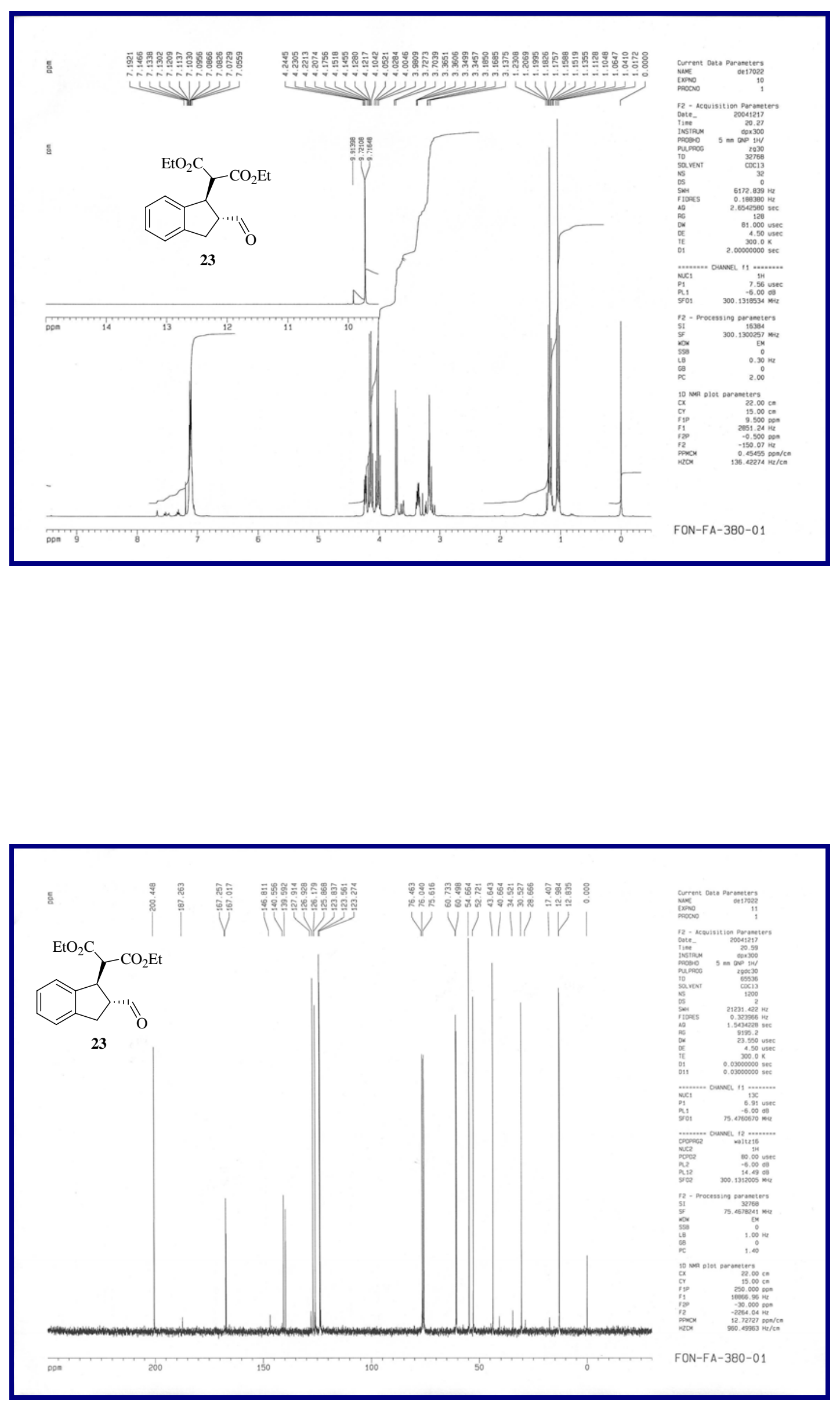

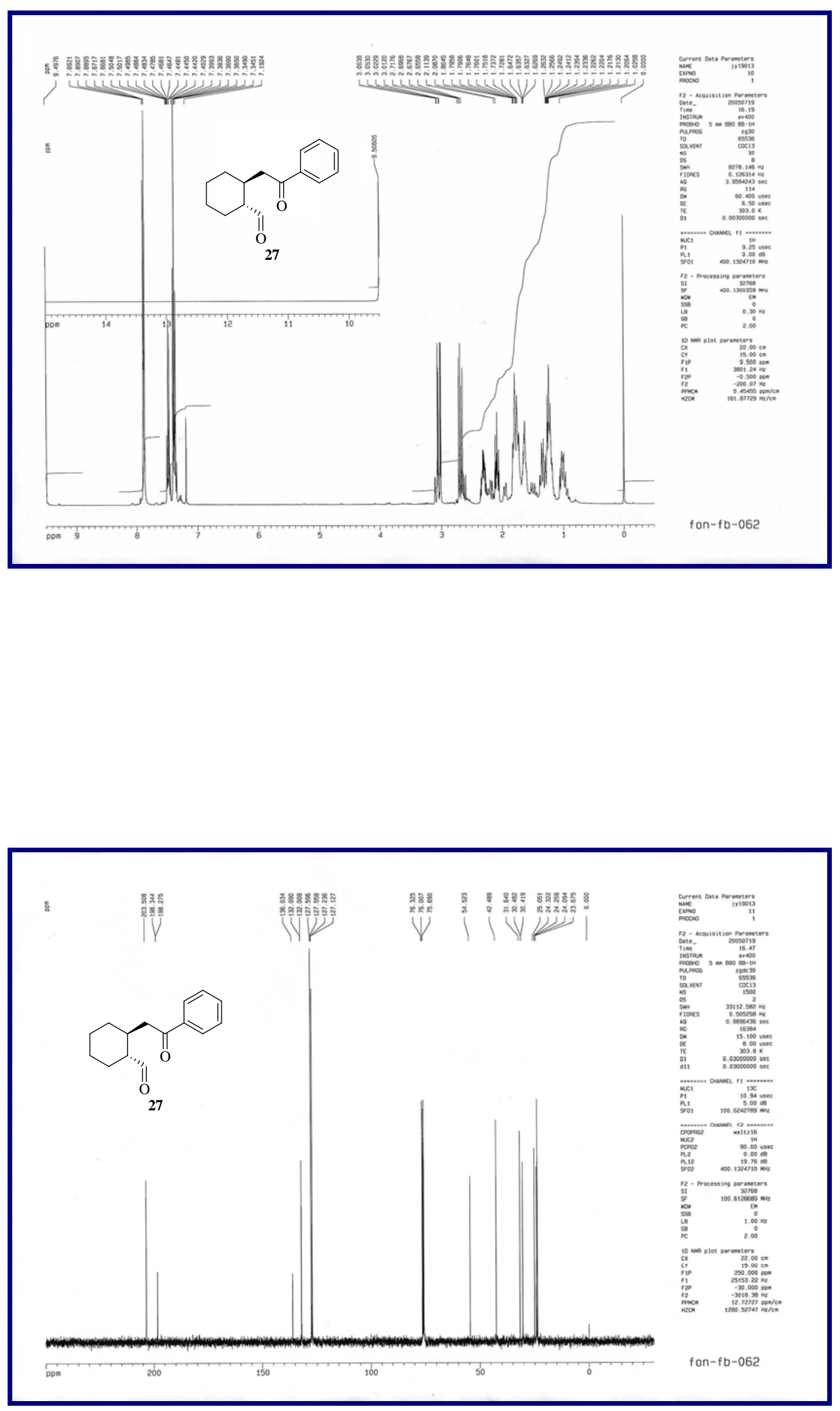

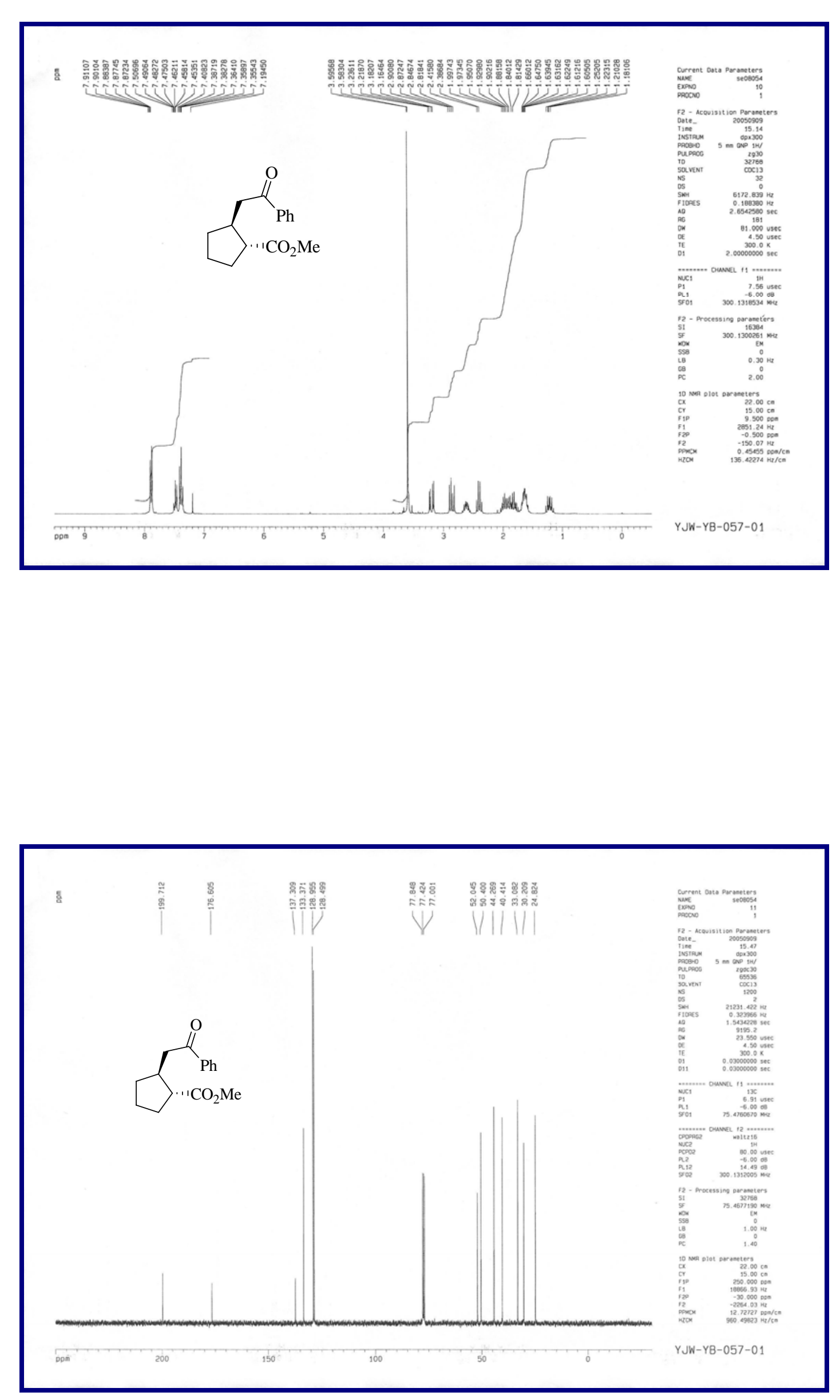